\title{
Stacked space-time densities: a geovisualisation approach to explore dynamics of space use over time
}

\author{
Authors: Urška Demšar ${ }^{1}$, Kevin Buchin ${ }^{2}$, E. Emiel van Loon $^{3}$ and Judy \\ Shamoun-Baranes ${ }^{3}$ \\ ${ }^{1}$ Centre for GeoInformatics, University of St Andrews, Scotland, UK, \\ urska.demsar@st-andrews.ac.uk \\ ${ }^{2}$ Department of Mathematics and Computer Science, Technical University Eindhoven, \\ the Netherlands,k.a.buchin@tue.nl \\ ${ }^{3}$ Computational Geo-ecology, Institute of Biodiversity and Ecosystem Dynamics \\ (IBED), University of Amsterdam, the Netherlands, [vanloon, shamoun]@uva.nl
}

\section{Corresponding author:}

Urška Demšar

Centre for GeoInformatics, School of Geography \& Geosciences

University of St Andrews, Irvine Building, North Street

St Andrews, Fife, Scotland

KY16 9AL

UK

urska.demsar@st-andrews.ac.uk, Tel: +44-1334-463890

\section{Acknowledgements}

Research presented in this paper is part of the collaboration under the COST (European Cooperation in Science and Technology) ICT Action IC0903, “Knowledge 
Discovery from Moving Objects (MOVE)” and facilitated by the Lorentz Center workshop on "Analysis and visualization of moving objects" (http://www.lorentzcenter.nl/lc/web/2011/453/info.php3?wsid=453). We thank Kees Camphuysen (NIOZ) and Arnold Gronert for all the field work and sharing expert knowledge related to the Lesser black backed gull project. The tracking infrastructure is facilitated by the BiG Grid infrastructure for eScience (www.biggrid.nl). The authors would like to thank Dr Jed Long and Dr Iain Dillingham from the Centre for Geoinformatics, University of St Andrews, for useful discussions in preparation of revisions of this paper. 


\title{
Stacked space-time densities: a geovisualisation approach to explore dynamics of space use over time
}

\begin{abstract}
:
Recent developments and ubiquitous use of global positioning devices have revolutionised movement ecology. Scientists are able to collect increasingly larger movement datasets at increasingly smaller spatial and temporal resolutions. These data consist of trajectories in space and time, represented as time series of measured locations for each tagged animal. Such data are analysed and visualised using methods for estimation of home range or utilisation distribution, which are often based on 2D kernel density in geographic space. These methods have been developed for much sparser and smaller datasets obtained through very high frequency (VHF) radio telemetry. They focus on the spatial distribution of measurement locations and ignore time and sequentiality of measurements. We present an alternative geovisualisation method for spatio-temporal aggregation of trajectories of tagged animals: stacked space-time densities. The method was developed to visually portray temporal changes in animal use of space using a volumetric display in a space-time cube. We describe the algorithm for calculation of stacked densities using four different decay functions, normally used in space use studies: linear decay, bisquare decay, Gaussian decay and Brownian decay. We present a case study, where we visualise trajectories of lesser black backed gulls, collected over 30 days. We demonstrate how the method can be used to evaluate temporal site fidelity of each bird through identification of two different temporal movement patterns in the stacked density volume: spatio-temporal hot spots and spatial-only hot spots.
\end{abstract}

\section{Keywords:}

Animal movement, space-time density, space-time cube, visual data exploration, home range estimation, utilisation distribution. 


\section{Introduction}

Recent ubiquitous accessibility and widespread use of global positioning devices using Global Navigation Satellite Systems (in particular the Global Positioning System, GPS) have enabled location-tracking on an unprecedented scale and produced large spatial data sets on moving objects. One of the scientific areas where these technological advances have brought a particularly significant advancement is movement ecology, which studies the movement of groups and individuals across different animal species, different scales of space and time and different levels of complexity [25].

Increased availability and decreased size of animal-tracking tags allows movement ecologists to collect large data sets on animal movement [7]. These data typically consist of trajectories in space and time: i.e. temporal sequences of measured locations for each tagged animal, as it moves through the physical world. Trajectory data are commonly used to describe the space use by the animal, and this is often done through derivation of home range or utilisation distribution using traditional methods [30]. However, these methods were developed in times when animal tracking data were collected using very high frequency (VHF) radio telemetry and were much more sparsely sampled. These methods are therefore not always appropriate for the increased size of tracking data sets, the dense spatial and/or temporal sampling and the complexity of the measured trajectories. Moreover, time is rarely taken into account in these approaches, which mainly focus on spatial/geographical distribution of data. One of the reasons for this may be the long-standing challenge of inclusion of time into spatio-temporal modelling and data representations in Geographic Information Science [36]. Alternative methods are required for analysis and visualisation of large and dense animal tracking data sets in order to identify patterns 
of movement - methods that take into consideration temporal information as well as the spatial (geographic) component.

In this paper we introduce stacked space-time densities of trajectories to visualise and visually identify movement patterns in space and time. The approach is inspired by the aggregated space-time density of trajectories within a space-time cube [13], but alters the density algorithm and combines it with home range/utilisation distribution principles in order to show the usage of space by an animal over time. The stacked densities volume in the space-time cube facilitates the following tasks in particular:

1) to visually identify spatial patterns in animal movement (for example, whether areas exist that are frequently visited by the animal) and

2) to explore temporal characteristics of animal movement and thus identify temporal changes in the use of geographic space (for example, does the animal visit the same point at the same time every day and stays there for a while, i.e. a spatio-temporal hot spot, or does it come and go often, but does not stay there and does so at different times, i.e. a spatial only hot spot).

This approach to visualisation can facilitate the identification of patterns in animal movement that may reoccur in space, time or both. For example, a researcher could distinguish between an area of intense use that is visited at the same time every day (spatio-temporal hotspot), or a geographic area used intensively but irregularly in time (spatial hotspot). Identification of such temporal patterns in movement has recently received much attention in movement ecology [42].

This is a primarily methodology paper in that it presents an algorithm for a new geovisualisation approach, which is not limited to animal trajectories in that it can be useful for any type of trajectory analysis where identification of spatio-temporal patterns is of interest. However, the approach was partially inspired by home range 
methods in movement ecology and therefore we chose to describe it in context of movement ecology and demonstrate its applicability on a case study with animal movement data.

The paper is structured as follows: first we introduce the concepts of home range and utilisation distribution and give a brief overview on spatial approaches to the derivation of these two quantities. We review approaches that focus only on space as well as approaches where time and sequentiality of trajectory points are taken into consideration. Then we present the algorithm for our new visualisation method - the stacked space-time densities - and the different decay functions used for calculating the stacked densities. We demonstrate how the method can be used to visually distinguish between two movement patterns: spatio-temporal hot spots vs. spatial-only hot spots. We do this by constructing two sets of simulated trajectories with the same spatial distribution of trajectory points but different temporal patterns of movement. This means that they are indistinguishable if considered only in 2D space and if only 2D density distribution is used to visualise the home range. We also evaluate the stacked densities algorithm against the geometrically optimal space-time density [13] with regards to identifiability of crucial visual elements (using Qualitative Result Inspection, QRI [29]) and computational efficiency (using Algorithm Performance measures [29]). We further apply the visualisation method to a case study of trajectories of lesser black backed gulls (Larus fuscus) to identify two different types of bird behaviour by distinguishing between spatio-temporal and spatial-only hotspots, similar to those in our synthetic example. Finally we conclude with some ideas for further method development and potential future research directions. 


\section{Home range and utilisation distribution in movement ecology}

In movement ecology, tracking data are often used to construct either the home range or the utilisation distribution of an animal. A home range is most often defined as a set of bounded areas used by an animal in the course of its normal activities (foraging, mating, caring for young). A utilisation distribution is a probability surface over a 2D area, describing the intensity of usage of a particular location by an individual [30, 56]. The two concepts are linked, since home range is often defined by an probability contour of the utilisation distribution surface for a certain value. Often a 0.95 probability is used, however the choice is subjective and may vary per study [20, 21]. A vast array of methods exists to estimate these two concepts. In this section we give a brief overview of spatial and spatio-temporal methodological issues only, since those are relevant to our extension into the 3D space-time cube. We characterise these methods according to the incorporation (or lack thereof) of the temporal aspect of animal movement into 1) static methods, 2) methods that take into consideration time non-sequentially and 3) methods that take into consideration sequentiality of movement (time between subsequent location measurements). We further point the interested reader towards other reviews that are more informative from the ecological perspective which is beyond the scope of this paper.

\subsection{Static methods}

Static methods for home range/utilisation distribution calculation derive these two concepts from measurements of the animal location. However, they do not consider the temporal aspect of the data. These methods were primarily developed for VHF telemetry data which are characterised by much lower and more irregular observation frequencies in comparison to modern GPS technology [30]. The oldest methods for 
the calculation of home ranges are geometric. The most prevalent geometric method is the Maximum Convex Polygon (MCP), which fits a convex hull to a certain percentage of observation points (usually 95\%) to define the home range. In spite of the rudimentary results, this method is still popular for its simplicity, ease of implementation and because it allows a relatively easy comparison between studies (e.g. [34] report that $68 \%$ of home range studies they reviewed used this method). Examples of more complex geometric hull-based methods include the k-nearest neighbour convex hull, i.e. a union of convex hulls associated with each point and its k-1 nearest points [19] and characteristic hull polygons, which consist of the densest areas in a Delaunay triangulation of observed points [14].

To calculate the utilisation distribution, a kernel density estimator is most commonly used to produce a probability surface from the location measurements of an animal. As with geometric methods described above, the temporal distribution of these points is not taken into account. Utilisation distribution methods normally use the standard two-dimensional point pattern kernel density estimation [50], which places a decay probability density function on each data point and then sums them up into a surface, thus producing an estimate of the probability of the animal being in each location. Most often, the decay function is a symmetric bivariate Gaussian kernel, although other decay functions are sometimes used [20]. As with the MCP, often the 95\% area of such utilisation distribution surface is used to define the home range of an individual [21]. There are numerous studies using some type of kernel density for utilisation distribution estimation (e.g. [5, 8, 20, 45, 56] to give a few selected examples from a very large body of literature spanning the last 25 years). Some reviews and comparative studies include [34], [55] and [30], the latter of which 
presents an evaluation of these traditional kernel density approaches for the new dense and large trajectory data sets.

\subsection{Methods taking into consideration time non-sequentially}

Static methods typically make (implicit) assumptions about the temporal distribution of the measurement points. Two common assumptions are regular observation rates and the independence of locations. While the second assumption is not explicitly temporal, it requires temporally sparse measurements because of the spatial autocorrelation of locations. Various methods address the case when these assumptions do not hold.

Observation rates might be irregular because of missing data, e.g. unsuccessful GPS fixes, or because of adaptive observation rates, e.g. observations only at daytime. [28] discuss approaches to correct for irregular observation rates for various home range models. Autocorrelation can be reduced by increasing the overall duration of observation and by decreasing the sampling rate $[18,41]$.

Usually, space use changes over time. In various studies the temporal dynamics are dealt with by aggregating location measurements over time. While sequentiality of points is not taken in consideration, the aggregation over different time scales provides a general impression of temporal patterns of the use of space. Temporal scale of aggregation varies with each study. For example, space use can be determined separately for daytime and night time or separately for different seasons of the year [53], different months [9] or years [52]. 


\subsection{Methods taking into consideration time via sequentiality of trajectory points}

Most of the methods described in the previous sub-sections use kernel density estimation, and thus explicitly or implicitly assume that the selected measurement points are (ideally) an independent sample drawn from a static probability distribution. The following methods take a more mechanistic perspective and are based on some model of movement, where the sequentiality of measurement points is taken into consideration.

Hengl et al. [24] connect consecutive measurement points by line segments, overlay the study area with a grid, and assign to each grid cell the parts of the line segments in the corresponding grid cell. This gives an estimate on the distance travelled within a grid cell. To obtain an estimate of time spent in a grid cell these distances are divided by estimated velocities. Decoupling computation of distances and velocities in this way allows incorporating environmental conditions (e.g. temperature) that influence the velocities.

Many sequential models are 2D kernel densities based on line segment kernels, i.e. in contrast with traditional point pattern kernel density (fig. 1a), kernels are not placed over each measurement point, but sequentially on each line segment (fig. 1b-1d). These segment kernels are then added into a two-dimensional surface. We list some examples of this type of kernels below.

Figure 1 somewhere here.

Long and Nelson [35] base their sequential line segment model on the space-time prisms from time geography [23]. They define a potential path area between two consecutive trajectory points as a projection of the space-time prism onto the 
geographical plane. This projection forms an ellipse, which is a set of all possible locations that a moving object might have traversed given the maximum possible velocity, the fixed start and end locations and the elapsed time between the two locations. Ellipses of all the trajectory segments are then combined into an estimation of the home range. Rather than producing a probability surface, their approach results in a uniform home range area, similar to geometric models.

An alternative time-geographic approach is presented by $[15,16]$, where a distanceweighted elliptical kernel, defined using the potential path area ellipse from the spacetime cube, is fitted to each pair of two consecutive points in the trajectory (fig. 1b). This results in a kernel that is symmetrical around the line segment of the trajectory. This methodology is generalised in [17] to a Delaunay triangulation of movement points for trajectories with irregular and infrequent sampling.

[33] adapt the elliptical line segment kernel by weighing its interior in the direction of movement (fig. 1d). I.e. the kernel function is a 2D projection of a cone centred on the first point of line segment and leaning towards the second point of line segment, where the inclination of the cone is calculated based on velocity and direction of movement.

The Brownian bridge movement model $[10,26]$ is based on a conditional random walk between every pair of successive measurement points. It also accounts for a location error at the measurement points. If the measurement error is assumed to be normally distributed then the location in any point in time is normally distributed. The mean of this distribution moves linearly between the locations of successive measurement points. The variance increases when moving away from a measurement point and then halfway to the next measurement point starts decreasing again (fig. 1c). This variance depends on the variance of the location error and on the mobility of the 
animal expressed by the Brownian motion variance parameter. [26] describe how to estimate this parameter using a maximum likelihood approach. In traditional models, the Brownian motion variance is assumed to be static along the entire trajectory. Recently, [32] introduced a dynamic version of Brownian bridges, where the motion variance is modelled to change over time.

Movement based kernel estimation [3] is an approach similar to Brownian bridges, which places additional points regularly sampled between consecutive measurement points and then places decay functions not only on the original measurements but also on the new points. [2] shows how this approach fits into a biased random walk model. [4] further extend this approach with calculating two additional spatial distributions: intensity distribution based on the mean resident time in each observed point and recursion distribution based on the number of visits to each point.

\section{Stacked space-time densities of trajectories}

Space-time density of trajectories is a generalisation of the 2D kernel density into 3D kernel density around polylines in the space-time cube [13]. The principle is based on geometric aggregation of movement data (i.e. trajectories) into a volume using a regular division of geographic space and time into voxels. This provides a spatiotemporal visualisation that displays movement patterns in a clearer way when compared to the space-time cube.

The standard space-time cube [23] can easily become cluttered and illegible when many trajectories are present in the display [31]. Therefore, some sort of aggregation of trajectory data is necessary for easier pattern recognition [1]. One important characteristic of the space-time density aggregation is that it preserves the property of the original space-time cube: to provide a visual illustration of continuity of 
movement in space and time, while at the same time allowing a trajectory-centred view of movement.

Space-time density of trajectories as a visualisation method was first introduced by [13]. It extends the principle of a sequential 2D line segment kernels (fig. 1b-1d) into a space-time cube, but generalises the kernel to follow the entire trajectory (fig. 1f), rather than building a separate kernel around each segment. While this methodology presents a geometrically optimal polyline density representation in $3 \mathrm{D}$, it has a disadvantage in that it is computationally complex due to calculation of distance in three dimensions [13]. In this paper, we address this issue by introducing a geometrically simpler model of 3D polyline density, the stacked space-time densities (fig. $1 \mathrm{~g}$ and $1 \mathrm{~h}$ ).

In stacked space-time densities, the temporal dimension is treated differently than the two geographic dimensions - this is in contrast to [13]. In particular, the decay is calculated for each moment in time, which in terms of implementation means for each horizontal voxel layer separately. This per-layer calculation represents the probability distribution of a moving object being in a certain location at one particular moment in time. Probability layers are then stacked one upon the other, to form the stacked space-time density volume (figs. $1 \mathrm{~g} \& \mathrm{~h}$ ). An algorithm for calculating this volume is presented below, including four different decay functions for probability estimation. It should be noted that the methodology presented here is not a special case of the methodology in [13]. There, kernels are calculated using distance from trajectory in 3D space and thus each kernel can be envisaged as a poly-cylindrical form following the trajectory, with spherical endings around end points (fig. 1f). Distance in this kernel is calculated perpendicularly to the trajectory, therefore its direction depends on the slope of the trajectory. This requires calculation of 3D distance between the 
centre of a given voxel and the entire trajectory, i.e. polyline (fig. 1f). The stackeddensities kernel however consists of a union of horizontal cylinders, one cylinder per voxel layer. In the case of temporally uniform kernel functions (linear, bisquare, Gaussian, fig. 1g), these cylinders are all of equal width. In the case of 3D Brownian kernel (fig. 1h), the width of each kernel depends on the location of the voxel layer between two consecutive trajectory points. The distance between each voxel and the trajectory is calculated only in the horizontal direction, i.e. in the temporal layer to which each voxel belongs (figs. 1g-h). This is therefore a 2D distance calculation between the centre of a given voxel and the intersection of the relevant line segment with the voxel layer of the given voxel. Geometrically, stacked kernels can be considered as special cases of the spherical 3D point pattern density (fig. 1e) [39] with three simplifications:

- $\quad$ uniform extent of the kernel in temporal direction (i.e. using cylinders instead of spheres),

- temporal extent set to zero (only consider kernel in one particular voxel layer) and

- $\quad$ rather than calculating kernels only at trajectory points (as [39]), we generate a separate cylindrical kernel at intersections of trajectory with each voxel layer. Trajectory kernel is then built as the union of all cylinders (figs. 1g-h).

We implemented the algorithm for calculation of the stacked space-time densities in Matlab, while the resulting density volumes were displayed in Voxler software, using volume rendering and isosurfaces. Volume rendering considers the volume as a medium of semi-transparent voxels, to which optical properties (colour and transparency) are assigned according to the volume value in each particular voxel [11]. Isosurfaces are $2 \mathrm{D}$ surfaces in the $3 \mathrm{D}$ volume such that all the points on the 
isosurface share the same value. Surfaces are displayed with different optical properties (colour, transparency) to give an impression of the distribution of one particular value inside the volume [22]. For more discussion on how to visualise space-time density volumes, see [13].

\subsection{Algorithm for stacked space-time densities}

The algorithm for calculating the stacked space-time densities takes as input a set of trajectories, each of which is given as a series of data points $(x, y, t)$, specifying the 2D geographic location (coordinates $x$ and $y$ ) at time $t$. Geographic location has to be specified on a plane (i.e. given in a projected coordinate system rather than as longitude and latitude). The resulting volume is assumed to have the same spatial resolution in the $\mathrm{x}$ and $\mathrm{y}$ directions (i.e. $\Delta x=\Delta y$ ). Temporal resolution of the volume, $\Delta t$, can differ from the spatial resolution. In the case study that we present in this paper, the total temporal range on the $\mathrm{z}$ axis is $24 \mathrm{~h}$ (given in seconds, i.e. $86400 \mathrm{~s}$ ) and the temporal resolution is 1000s. These values for temporal range and resolution were chosen as we were interested in daily patterns of animal movement. The method however is general and allows for any kind of temporal extent and resolution. For example, the temporal range could be a month and the resolution a day, or the range could be a year and a resolution a week or a day, depending on the purpose of the ecological study and the temporal acquisition density of data available (i.e. temporal sampling rate).

The algorithm reads each trajectory sequentially and subsamples the points to the chosen temporal resolution. In this context we consider a trajectory to be the sequence of locations describing movement of one object over the entire temporal period (i.e. 24h). For each line segment of the subsampled trajectory, the algorithm finds all the 
temporal voxel levels that belong to this particular line segment and calculates the density for each temporal voxel level. This is done according to a selected decay function, where decay is based on geographic distance of each voxel from the central point on the trajectory at that particular moment in time. Decay functions are described in detail in the next sub-section. They are all given as 2D probability distributions over $x$ and $y$ dimensions, i.e. given the central point on the trajectory at a certain moment in time (voxel level), they describe the probability of the moving object being in one particular voxel at that particular moment in time. These probabilities are then stacked one upon the other to produce the stacked trajectory density volume. Finally, stacked trajectory density volumes are added up for all trajectories and normalised by the number of trajectories, which results in the final stacked total density volume.

The pseudo code of the algorithm is given in the appendix.

\subsection{Different types of decay functions}

As mentioned above, each decay function $f$ is a function of the geographic distance from the subsampled trajectory at a certain moment in time. We use a decay function which is symmetrical around the $z$ axis (i.e. the same in the $x$ and $y$ directions). Therefore, for simplicity, we look at geographic distance only in the direction of the $x$ axis and denote it by $x$. In our notation, the decay function $f$ therefore becomes a function of $x$, i.e. $f(x)$.

We implemented four different decay functions: linear, bisquare, Gaussian and Brownian. There is some evidence for $2 \mathrm{D}$ point density that the choice of kernel function does not affect the general visual pattern [50, p. 42-43]. While for our 3D stacked densities we expect a similar result for the first three decay functions (linear, bisquare and Gaussian), we further expect that the Brownian decay will visually 
emphasise the certainty of location in the measured points and uncertainty of movement between the points - something that will not be directly observable in the pattern produced with the first three functions.

Fig. 2 shows these functions and the resulting stacked space-time density for each function type.

Figure 2 somewhere here.

Each of the decay functions $z=f(x)$ is rotated around the $z$ axis to produce a surface that is used to calculate decay around the central point of the trajectory at one particular voxel layer. Using these surfaces means that we can describe the resulting density value in each voxel of this layer as the probability of the moving object being in that particular geographic location at that particular time. To achieve this, the surfaces need to represent 2D dimensional probability distributions and therefore need to fulfil the requirement that the volume underneath each surface is equal to 1 . We mention this condition specifically since sometimes in similar derivations [27], a 1D probability distribution is used instead, although it does not satisfy the volumetric requirement.

Linear and bisquare decay functions are given by the formulas (1) and (2) respectively:

$$
\begin{gathered}
z_{\text {Linear }}(x)=\left\{\begin{aligned}
a \cdot \frac{(h-x)}{h} ; & \text { if } x \leq h \\
0 & ; \text { if } x>h
\end{aligned}\right. \\
z_{\text {Bisquare }}(x)=\left\{\begin{aligned}
a \cdot \frac{\left(x^{2}-h^{2}\right)^{2}}{h^{4}} & ; \text { if } x \leq h \\
0 & ; \text { if } x>h
\end{aligned}\right.
\end{gathered}
$$


Here, $h$ denotes the user-specified bandwidth and $a$ the height of the linear triangle (fig. 2a) or the bisquare bell (fig. 2b). In this particular derivation we do not discuss how the specific bandwidth $h$ is selected and assume that some study-specific method has been used to select an appropriate bandwidth size. Optimal bandwidth selection is a large issue not only in ecology but any time when kernel density estimation is used. In standard 2D kernel density estimation, the size of the bandwidth determines the level of smoothing of any point pattern in 2D: a too-small bandwidth emphasises individual points, while a too-large one over-smoothes the pattern [50]. A similar bandwidth-choice effect may be observed in 3D around polylines. In multivariate visualisation, optimisation algorithms are typically employed to determine the best bandwidth - in this respect we point the reader to [38] for more details.

The rotation of the linear decay function around $z$ axis produces a cone with the top at the central point of the trajectory and a linear decline to a circle with diameter $h$. Everywhere else the surface is equal to zero. Rotation of the bisquare decay function around the $z$ axis produces a bell-shaped surface, which also reaches zero at the circle with diameter $h$. In both cases, the height $a$ is determined so that the volume under the rotated surface (i.e. the cone or the bell) is equal to 1 , to satisfy the condition for these being $2 \mathrm{D}$ probability distributions. In both cases this height has to be equal to $a=3 / \pi h^{2}$. Therefore we use the following two functions in density calculations:

$$
\begin{gathered}
z_{\text {Linear }}(x)=\left\{\begin{aligned}
\frac{3}{\pi h^{2}} \cdot \frac{(h-x)}{h} ; & \text { if } x \leq h \\
0 & ; \text { if } x>h
\end{aligned}\right. \\
\text { And } \\
z_{\text {Bisquare }}(x)=\left\{\begin{aligned}
\frac{3}{\pi h^{2}} \cdot \frac{\left(x^{2}-h^{2}\right)^{2}}{h^{4}}, & ; \text { if } x \leq h \\
0 & ; \text { if } x>h
\end{aligned}\right.
\end{gathered}
$$

The Gaussian decay function (fig. 2c) uses the standard 2D Gaussian distribution ${ }^{1}$ :

\footnotetext{
${ }^{1}$ Note that [26, 27] present the formula with a square root in the denominator, which corresponds to a $1 \mathrm{D}$ (not 2D, as required) Gaussian distribution. Their derivations are not affected by this.
} 


$$
z\left(x, \mu, \sigma^{2}\right)=\frac{1}{2 \pi \sigma^{2}} \cdot e^{-\frac{(x-\mu)^{2}}{2 \sigma^{2}}}
$$

Here, $\mu$ is the mean and $\sigma^{2}$ the variance that describes the mobility of the object. In our case the function needs to be centred on the trajectory, therefore we set $\mu=0$. This results in the following formula for Gaussian decay:

$$
z_{\text {Gaussian }}(x)=\frac{1}{2 \pi \sigma^{2}} \cdot e^{-\frac{x^{2}}{2 \sigma^{2}}}
$$

In this case, $\sigma^{2}$ is a constant and does not depend on the position on the trajectory. For this experiment we set it to $\sigma^{2}=(h / 2)^{2}$, since the purpose of this paper is primarily to develop the visualisation method and this particular value allowed us to visually compare resulting densities with the ones calculated using other decay functions. Here $h$ is the user defined bandwidth, as with linear and bisquare functions above. The actual value of this parameter in home range studies depends on animal mobility and therefore varies with each animal species under observation [26].

The rotation of the Gaussian decay function also produces a surface in the form of a bell (fig. 2c), which is similar to the bisquare one (fig. 2b). However, this bell surface never reaches zero, but falls towards zero in all directions. We also do not have to calculate the height of the bell of this function to ensure that it satisfies the requirement of the volume beneath the rotated surface to be 1 , since we have started with a 2D probability distribution (eq. 4) and this condition is therefore already satisfied.

One property of the described decay functions (linear, bisquare, Gaussian) is that they only depend on externally-set bandwidth $h$, which is constant for entire trajectory. Distance decay is therefore the same regardless of the position on the trajectory. In figures 2a-2c this is shown by the fact that the isosurfaces for constant values of 
space-time density, while spaced differently for each type of decay function, are parallel to the trajectory. This however does not consider uncertainty in movement between two consecutive measurement points. To address this uncertainty we introduced the fourth type of decay using the concept of Brownian bridges [10, 26, 27].

Brownian decay is a concept based on the stochastic description of animal movement, $[10,26]$, as described in the previous section. Here, the probability of the moving object being in a certain point depends on the start and end locations (i.e. on two consecutive measurement points), the elapsed time $t$ since the object left the start location and the speed of movement (described by the time the object needed to go from start to end location, marked as $T$ ). In terms of home range estimation, Brownian decay is modelled as Gaussian decay which varies not only with distance from trajectory, but also with $t$ and $T$ (fig. 2 d), i.e. $z=f(x, t, T)$. This means that at each point on the trajectory we take the Gaussian decay function (eq. 5), and set the variance $\sigma^{2}$ to vary with $t$ and $T$. The way to model this dependency is given by [10] as:

$$
\sigma^{2}(t, T)=\sigma_{1}^{2} \cdot(1-t) \cdot t \cdot T+\sigma_{2}^{2} \cdot T \cdot\left((1-t)^{2}+t^{2}\right)
$$

Here, $\sigma_{1}^{2}$ and $\sigma_{2}^{2}$ are constants describing two different aspects of uncertainty in the measurement of movement [10]. $\sigma_{1}^{2}$ is the variance of movement along the trajectory (the same as $\sigma^{2}$ in Gaussian decay above). $\sigma_{2}^{2}$ is the variance of the distribution that reflects the uncertainty of the object being at start and end locations - i.e. the uncertainty of the location measurement. $\sigma^{2}$ is related to animal mobility and is a feature of a particular animal species under observation, while $\sigma^{2}{ }_{2}$ depends on properties of the measurement equipment [26]. For the purposes of this paper, we set the values to the following ( $h$ being the user defined bandwidth, as in linear and bisquare densities above): 


$$
\sigma_{1}^{2}=\left(\frac{h}{2}\right)^{2}, \sigma_{2}^{2}=\frac{2 \sigma_{1}^{2}}{5}
$$

However, as discussed above, values of both these two parameters depend on animal mobility and the measurement equipment and how these values are related to the bandwidth $h$ and to each other should be taken into consideration in each particular experiment, depending on the animal species under investigation.

\section{Why choose 3D over 2D? Identifying temporal patterns in movement data}

One of the persisting questions in movement ecology are temporal patterns in space use. Animal use their environment unevenly by spending more time in favourable locations or revisit such locations more frequently than others and it is of specific interest to ecologists to find out the role of time in this heterogeneous behaviour [4, 42]. Most methods for home range estimation however are linked to two geographic dimensions and therefore do not allow visual discovery of temporal patterns very easily. This is where incorporating the space-time cube principle into visualisation for home range dynamics is needed to support distinction between different types of patterns. In this section we show how traditional 2D density masks the difference in two temporally different visitation patterns to a central location and how these patterns are immediately obvious when using 3D densities.

To evaluate the 2D kernels vs. 3D kernels, we generated two synthetic sets of trajectories and calculated their respective 2D and 3D densities (figure 3). Each set contained the same number of trajectories. Each corresponding trajectory in both sets contained the same number of points, which had an identical spatial distribution. That is, in both cases, each trajectory described movement from the border of the study area $([0,10] \mathrm{x}[0,10]$ rectangle in the $\mathrm{x}-\mathrm{y}$ plane) towards the central point and back. 
Corresponding trajectories in figs. 3a, 3b and 3c are coloured the same. However, in each case, the trajectories exhibited a different temporal pattern of movement.

Set A consisted of four trajectories that first moved to a central point of the study area and then outwards from this point, but where each of them reached the central point at a different moment in time (fig. 3a). These trajectories represent a spatial-only hotspot, i.e. an area which the moving objects frequent, but never reach this point at the same time.

Set B consisted of four trajectories that met at the central point at the same time (fig. 3b) and thus demonstrated spatio-temporal convergence of trajectories. In this case, the central area represents a spatio-temporal hotspot.

\section{Figure 3 somewhere here.}

In both cases, A and B, the trajectory points produce an identical spatial pattern in two geographic dimensions (fig. 3c). This means that if a standard 2D kernel density is used for estimation of home range on either data set, the result is an identical surface (fig. 3d). Specifically, fig. 3d shows a traditional 2D kernel density surface, calculated on either of the two trajectory sets, using the bisquare decay point kernels (as those illustrated in fig. 1a), 2D raster resolution of 0.1 and bandwidth size of 0.8 . As noted, based on such a 2D-only case, it is not possible to distinguish between sets A and B, since the only distinguishing factor between A and B is the difference in the temporal pattern, which 2D density ignores.

Figures 3e and 3f show stacked space-time densities of cases A and B respectively, calculated with the same parameters as the 2D density in figure $3 \mathrm{~d}$. We use 3D bisquare stacked density kernel (as in fig. 1g), voxel resolution of 0.1 and bandwidth 
size of 0.8 . The 3D volumetric patterns are clearly different and illustrate the difference between a spatial-only and a spatio-temporal hotspot - something that is not identifiable in the 2D density of the same two data sets (fig. 3d). Stacked spacetime densities therefore allow distinguishing between areas where objects congregate at the same time and others where they visit only at different times.

\section{Comparison of 3D densities: space-time density and stacked densities}

In order to evaluate the two 3D density algorithms against each other, we developed a specific evaluation procedure (fig. 4). In ecology and statistics, different types of 2D densities are often compared by using an artificial data set, calculating different density surfaces on the same data and then evaluating differences between surfaces through calculation of various error measures [20]. This is illustrated as the first purple arrow (“Statistical evaluation”) between two density fields in figure 4. However, this kind of comparison, is only possible for multivariate densities if all dimensions are treated in the same way across the different algorithms that are to be compared [54]. Our two density algorithms treat the temporal dimension differently and therefore we cannot compare them in this way.

Figure 4 somewhere here.

An alternative method of comparison that may be considered is common in volumetric visualisation: there, different types of volume rendering (which we use to display our density fields) are often compared through calculation of efficiency measures, such as depth coherence or distinguishability of features [57]. The second purple arrow ("Volumetric visualisation evaluation”) indicates this possibility in fig. 4. However, we use the same volume rendering parameters for both density fields, 
hence with respect to these factors there is no difference between our two algorithms and these measures are not applicable in our case either.

Instead, we employ evaluation measures from information visualisation [29] and use the two most prevalent evaluation methodologies in the visualisation community: Qualitative Result Inspection (QRI) and Algorithm Performance (AP). QRI is a method where a certain visualisation methodology is used to produce an image that demonstrates how something that could not be seen before can now be seen with the new method. An extensive review of information visualisation studies [29] found that QRI was used in 95\% of all cases. An example of this methodology used for 2D trajectory density can be found in [49]. AP was the second most frequent evaluation method, used in 35\% of studies. It is a quantitative measure of algorithm speed, which is linked to computational complexity.

For QRI in our case, we calculated space-time density [13] and stacked densities on the two data sets from section 4 and visually compared the two results. Stacked density volumes for these two data sets are shown in figures 3e and 3f, while spacetime densities for the same two data sets are shown in figures $5 \mathrm{a}$ and $5 \mathrm{~b}$ respectively. All four density fields were in the same range $\left([0,10]^{3}\right)$, volumes were of the same size (100x100x100, voxel size 0.1$)$ and kernel size in all cases was 0.5 (5 pixels on each side of trajectory). We used the same kernel size in both cases (linear kernel). It is clear that figures 3e and 5a show a similar picture where non-interaction of the four trajectories can be observed. In figures $3 f$ and $5 b$, the spatio-temporal hotspot is also clearly identifiable. The two 3D density algorithms therefore both allow visual identification of patterns of interest to ecologists.

Figure 5 somewhere here. 
In terms of AP, we first calculated the asymptotic worst-case running times of both algorithms. The asymptotic running time of the geometrically optimal space-time density [13] is in $\mathrm{O}(n \times m \times v)$, where $n$ is the number of trajectories, $m$ the length of the longest trajectory (number of measurement points in the longest trajectory) and $v$ the number of voxels in the density volume (and thus equal to $v_{x} \mathrm{x} v_{y} \mathrm{x} v_{t}$ ). This results in long calculation times for data sets with a large number of densely sampled trajectories and when density volumes are required to have a detailed spatial and temporal resolution and thus many voxels in each of the three dimensions (i.e. large $\left.v_{x}, v_{y}, v_{t}\right)$. In contrast, the stacked space-time densities present a computationally less demanding solution, as the asymptotic running time of the algorithm is in $\mathrm{O}(n \times v)$.

To demonstrate the change in algorithm speeds, we set up an experiment where we again used case A and case B data from the previous section. We subsampled both data sets so that trajectory length for each of the four trajectories in the data set was either 50 or 100 (i.e. there were 50 or 100 points on each trajectory). We used the same data range for all cases, but set up two different volume sizes, either 10x10x10 or 100x100x100. We used the same kernel type (linear) and the same kernel size in all cases. With all parameters kept the same, table 1 shows the difference in processing times between the two algorithms. The much reduced times of stacked densities clearly demonstrate the difference in processing times, in line with the asymptotic worst-case running times.

Table 1 somewhere here. 
The results of QRI and AP indicate that stacking the densities temporally vs. using a geometrically optimal solution speeds up the calculation significantly while producing a visually adequate density volume for identification of spatio-temporal patterns of interest.

\section{Case study: using stacked space-time densities to visually explore movement patterns of lesser black backed gulls}

As part of a long-term field study, the movement of lesser black backed gulls is monitored and analysed to better understand the foraging ecology of this species and ultimately link this to breeding success [12]. Currently, the time budgets of individuals are quantified and areas where they potentially feed are located. Lesser black backed gulls may exhibit inter-individual differences in behaviour due to individual specialisation in foraging behaviour, spatial and temporal variations in resource availability, influences from weather and breeding status.

Individuals may show different levels of site fidelity, revisiting the same area for short periods of time on numerous occasions, or make rare long visits to specific locations (especially when the resource is predictable and stable). In a spatial only context, both types of behaviour could result in similar space use patterns. Identifying temporal differences in spatial patterns and regularity of visiting a given area, in combination with the total time spent at that location may therefore provide interesting and new insights with respect to foraging strategy and behavioural ecology. 


\subsection{Data description}

The data for this study were provided for an interdisciplinary workshop [47] and comprise tracks from 1 June 2010 - 30 June 2010 of four adult male and four adult female lesser black-backed gulls breeding on Texel, the Netherlands (fig. 6). Data were collected using the UvA Bird Tracking System (http://www.uva-bits.nl/, [6]). Nest-locations for each of the individuals are also known. Considering trips with the nest as start and endpoint, and moving at least $3 \mathrm{~km}$ away from the nest, on average 467 trips were made per individual, with a mean trip duration of 5.3 hours, the mean distance along the trajectory per trip was $69.0 \mathrm{~km}$, and the mean maximum Euclidian distance from the nest was $24.3 \mathrm{~km}$.

Figure 6 somewhere here.

Figure 4 shows observed location points of the two birds that we selected for density calculation in this paper, no. 298 and no. 311 (figs. 4a and 4b). These are both male birds and we were interested in temporal patterns in their movement indicating site fidelity to locations away from the nest. For this purpose, we excluded data points in the nest and its immediate vicinity (150m), as shown in figs. 6c and 6d. The distance of $150 \mathrm{~m}$ was chosen to delineate start and end of trips (i.e. when the bird moved far enough from the nest for the movement to be considered a trip from the nest) as well as for correction for central-place foraging behaviour, which we discuss in the next section.

\subsection{Calculating the densities and correction for central-place foraging behaviour}

In this section we describe the procedure for density calculation that takes into account a particular type of animal behaviour: central-place foraging [40, cited in 51, 
p. 277]. This behaviour means that during each day, the animal makes several backand-forth foraging trips from a central location, each time returning back to that location (to e.g. bring food to the young in the nest). This behaviour introduces a geographic bias into the use of space, since it is more likely for the animal to be located in the central location and its immediate neighbourhood than anywhere else at any observed time. As we are interested in wider movements of the gulls and not in their movement in nest area, here we adjust our calculations for this behaviour by removing data points from the nest and its surroundings and further discuss other more complex solutions in the conclusions.

We calculated the stacked space-time densities for the two selected birds (no. 298 and no. 311) using the same spatial and temporal extent, to produce comparable density volumes. The geographic area covered by the trajectories of both birds measured $100 \mathrm{~km} \times 100 \mathrm{~km}$ in E-W and N-S directions. Temporal extent was $24 \mathrm{~h}=86400 \mathrm{~s}$, starting from $0 \mathrm{~h}$ at the bottom and continuing towards $24 \mathrm{~h}$ at the top. Spatial resolution in both east-west and north-south direction was $1000 \mathrm{~m}$ and temporal resolution 1000s (16min 40s), which resulted in a density volume of 100 x 100 x 87 voxels. Kernel size (bandwidth $h$ ) was set to $5000 \mathrm{~m}$. The temporal range of $24 \mathrm{~h}$ was chosen (as opposed to showing the entire month of data on the $\mathrm{z}$ axis) to be able to identify daily behavioural patterns of birds (if any) and their potential repetition over the study period. The temporal resolution of 87 voxels per day on the other hand provided manageable computational complexity while still ensuring enough details to be able to identify behavioural patterns in movement. Figure 7 shows the resulting space-time density for all four decay functions for bird 298, shown with volume rendering. 


\section{Figure 7 somewhere here.}

If we compare the four decay functions visually, they all give a similar picture of the movement of this bird. There are certain differences though, for example, linear decay density has crisper borders than the others. Bisquare decay emphasises higher density values (more and brighter red in the temporal columns, fig. 7b). Gaussian density fades slower than the others and never reaches zero (fig. 7c). In Brownian decay uncertainty is taken into consideration, which visually results in dense areas around measurement points (fig. 7d). These dense areas and areas of decreasing and increasing density in-between are the 3D visual representations of the Brownian bridges concept.

\section{Figure 8 somewhere here.}

One difficulty that we encountered at this point in the process was the fact that lesser black backed gulls are central-place foragers, meaning that they return to a central point (in this case a nest situated within a breeding colony), sometimes several times a day, as well as spend a significant proportion of time at the nest. This means that a significant proportion of trajectory points for each gull were collected while the animal was in the breeding colony where it does not forage. Consequently these points do not describe the movements we are interested in. In our case, over 30 days of measurements, bird 298 had 1774 points in the nest out of 4161 points in total (42.6 \%) and bird 311 had 2052 nest points out of 4096 points in total (50\%). Since the temporal sampling rate of original data was not constant over the data acquisition period, these percentages do not equal percentage of time spent in the colony. However, if these points are included in the space-time density calculation, the 
resulting volume has very high values in a temporal column over the nest. This column is visually very prominent, while the density over the rest of the geographic area is less clear, since the density values are lower and therefore volume rendering method assigns higher transparency to these areas (fig. 8a). To avoid this effect we removed points within $150 \mathrm{~m}$ from the nest (figs. 6c and 6d) and calculated spacetime densities on the remaining points only (fig. 8b). The area around the nest is still represented as a temporal column, since the bird has to pass this area when moving in and out of the nest. While bird 311 is at the nest predominantly during the night (hence more points around the nest are removed during the night) a consequence is that especially the movements in and out of the colony during the day become more visible in fig. 8b. By removing the nest-effects, figure $6 \mathrm{~b}$ shows more clearly than fig. 8a, that bird 311 moves much less at night. In addition, the pattern of one key area outside the nest often revisited becomes more obvious once nest effects are removed.

\subsection{Results: identifying movement patterns from density volumes}

Figures 9 and 10 show stacked space-time density volumes for birds 298 and 311. In both cases, densities are shown with direct volume rendering using perspective view (figs. 9a, 9b, 10a and 10b) and orthographic projections from the south or east (the front or the side of the volume cube) and the top (figs. 9c, 9d, 10c and 10d) using two different colour schemes. We also show the two density volumes with isosurfaces in perspective view (figs. 9e and 10e).

Figure 9 somewhere here.

Figure 10 somewhere here. 
These volumes identify the daily movements of each of the birds, aggregated in a space-time cube over the 30 days for which we had data. In these cubes, the $x-y$ plane represents the geographic plane and the $z$ axis the temporal axis over the 24h range. The value of density represents the probability of the animal being in a particular area at a particular time of day, which gives a visual impression of daily patterns of the space use.

In addition, particular types of visual patterns in the density volume can be used to explore the properties of daily movement of each animal. In these two examples, we can identify a prevalent temporal column in densities of both birds in the area surrounding the nest. This column stretches across the entire 24 hours (i.e. across the entire extent of the $z$ axis) and represents movement in the vicinity of nest area, which is expected for a central place forager during the breeding season. This column is an indication of when, during the course of 24 hours, an individual moves to and from the nest.

Another type of pattern of interest are hot spots, either spatio-temporal (a place where a bird repeatedly visits at the same time each day and stays for a while) or spatial-only (bird returns to this place often, but there is no temporal pattern to the visits). Visual exploration of density volumes can help distinguish between the two, as demonstrated on simulated data in the previous section. Bird 298 has one clear spatio-temporal hot spot with high density values that are in close proximity to each other in both space and time (figs. 9a-9d, indicated with an arrow in 9d), which is also shown as an isosurface (fig. 9e). This hot spot represents a spatio-temporal convergence of trajectories, which in the context of the bird movement means that the bird tends to visit this place often (see arrow in fig. 9d), comes there mostly in the early hours of 
the day and stays there for a while (fig. 9c). This pattern is similar to the pattern in case B of simulated data (figs. 3b and 3f).

In contrast, bird 311 has a spatial-only hot spot. It is a spot that the bird visits often, best seen from the view from top (fig. 10d), however, there is no pronounced temporal pattern over this spot as with bird 298. Visits are sporadic and short and occur at any time of day - this can be best seen by a light-blue to dark-blue vertical area to the left of the nest column in the view from the east (fig. 10c). This means that the bird revisits this place, but does not stay there for a longer time. The same pattern can be seen in the isosurface (fig. 10e), where there is no uninterrupted continuous isosurface of high density over the spot - instead there are several smaller high density areas that each represent one or a few temporal visits to this particular location. This pattern corresponds to case A in the simulation (figs. 3a and 3e).

The next step is to explore the possible reason for visiting these sites and the potential activities that take place there by adding the context and knowledge about the area. The spatial-only hot spot visited by bird 311 is a water purification plant, visited by gulls for preening and resting (A. Gronert, personal communication), whereas the spatio-temporal hot spot of gull 298 is an agricultural area where gulls potentially forage and roost. These and similar spatial and spatio-temporal hot spot patterns could be a useful tool for identification of patterns of periodicity in recursions to a particular location, a temporal characteristic of animal movement [42]. Such observations could be further confirmed using either visual observations or additional sensor observations (e.g. accelerometer [48]). 


\section{Conclusions}

In this paper we presented stacked space-time densities as a method for visualising movement patterns and temporal dynamics of space utilisation in a data set of animal trajectories. The method is based on a concept of space-time cube, thereby preserving the fundamental linkage between space and time, as introduced in time geography. The aim is to give a visual impression of temporal dynamics of space use via volumetric aggregation of trajectory data. We anticipate that the method could be of use in animal ecology, where animal tracking is producing unprecedented amounts of data due to development of new tracking technologies. We see this approach as primarily useful for the initial visual exploration of data and building hypotheses about the spatio-temporal aspects of animal behaviour, which are not identifiable via traditional 2D home range/utilisation distribution mapping, as demonstrated in simulated cases A and B and real bird examples in the previous sections. Ecologists can then further verify or reject these hypotheses using e.g. resource selection models [37].

In the paper most of the examples are shown using volume rendering of the density volume. In terms of $2 \mathrm{D}$ home range methods this is equivalent to showing the utilisation distribution surface as a raster map. In order to delineate home range from utilisation distribution, typically some contour (isoline) of this surface is used. In our 3D density volume, the equivalent would be to display an isosurface of the volume (as in figs. 7e and 8e). In contrast with the 2D case, where the isoline delineates the overall home range, the respective isosurface in the 3D volume would show the changes of the home range over time, thus allowing visual identification of temporal patterns in movement, such as periodicity of visits and their temporal extension. 
From the algorithmic point of view of 3D density calculation we note that our approach includes geometrically simple kernels, which are calculated per voxel layer. In cases where kernel sizes are independent of time (using linear, bisquare and Gaussian kernels, fig. 1g), this implies that probabilities of animal being in a certain location at a particular time are only connected to each other by the position of the kernels, which are centred around the interpolated line segment in 3D space. In case of Brownian bridges there is a further implicit inclusion of time into calculation in that kernel sizes depend on the position on the line segment at each specific moment in time. More complex kernels that model probabilities through time are possible, such as for example instead of a cylinder creating a half-spherical, spherical or elliptical kernel at each voxel layer which extends into previous or future times. More complexity could also be added by making the kernel non-symmetrical in 3D, but space probabilities within the kernel based on movement direction and velocity (as an extension of [33] into 3D). These variations present a future challenge for 3D visualisations of movement.

While developing the approach, we encountered certain issues, on which we speculate in the rest of this section and point out potential future solutions. One such issue is that in our case study, we use data from a central-place foraging animal. This type of behaviour introduces certain biases in the use of space and is a well-known modelling problem [44]. In general, methods to estimate home range are based on comparison with null model of animal movement, which assumes that there is no spatial context for habitat use [44]. Our visualisation method is of this type - nowhere in the calculation of stacked space-time densities do we assume that there is a different expectation of space use to anywhere else. However, as mentioned above, lesser black backed gulls exhibit central-place foraging behaviour. This also introduces a 
geographic bias to movement analysis, in that birds need to cross areas close to the nest more often than those further away. Since the aim of this paper was to develop the new visualisation method that is general for all types of movement (i.e. not specifically aimed a central-place foraging animals), we settled for a relatively simple solution by removing the nest points from the trajectory dataset. We are aware that this is a rather basic attempt and while removing the nests did result in avoiding the visual domination of the nest as a temporal column in the density volume, this does not solve the geographic bias of exiting and entering the nest, therefore making it more likely for this area to have higher probability of presence. In habitat use studies, this problem is sometimes solved by subtracting a null model of distance use that takes into consideration declining resource use with distance [44]. I.e. the expected probability of the animal being in a certain location decreases not only with the distance from each measured location (trajectory point) but also with the distance from the central place. This works well for space utilisation distribution via 2D density estimation, and we are currently considering a generalisation into 3D spacetime density using a similar principle.

Another potential development that we see is to use our ideas of calculating densities in three dimensions to extend the home range/utilisation distribution methodology into real 3D space (using elevation as the third dimension). This is particularly relevant for animals that move in the air or in water changing their vertical distribution in relation to environmental factors [46, 43]. We expect that it would be possible to calculate the use of space by the bird in all three dimensions as a volume in a similar way as in a space-time cube, but where time would now represent the fourth dimension and would therefore have to be shown in a different way, perhaps with a slider or animation. 
Another development that may be of interest is linked to the concept of Brownian decay function. In our implementation, the form of the Brownian bridges depends on two types of uncertainty: uncertainty in measurement of location and uncertainty in animal movement. These two quantities are considered as constant, regardless of where on the trajectory we are (eq. 6 and 7). However, the uncertainty in movement may not be the same everywhere. This means that the parameter that describes this uncertainty $\left(\sigma_{1}^{2}\right)$ may also vary over total time, depending on the type of movement of the animal [32]. For example, different flight types of a bird (e.g. smooth soaring vs. flapping, where more tortuosity is present in the observed trajectory) may result in a different values of $\sigma_{1}^{2}$, which means that at certain types of movement, the Brownian bridge would be wider or thinner than at other times. Such considerations could be included in the density calculation.

In addition, uncertainty in location measurement $\left(\sigma^{2}{ }_{2}\right)$ may also depend on several factors. While precision and accuracy of the measurement equipment are often considered as constant throughout the measurement process, positioning uncertainty can also be affected by spatially and temporally varying factors such as land cover, topography, atmospheric conditions and coverage of positioning satellites at each measurement location. If this uncertainty has a large impact on the results and can be reliably estimated, it should be incorporated in Brownian decay modelling.

\section{Appendix - algorithm for calculation of stacked space-time densities}

This appendix presents the pseudo code of our algorithm.

Input: a set of movement trajectories, volume resolution (in $x, y, t$ ) and bandwidth size. 


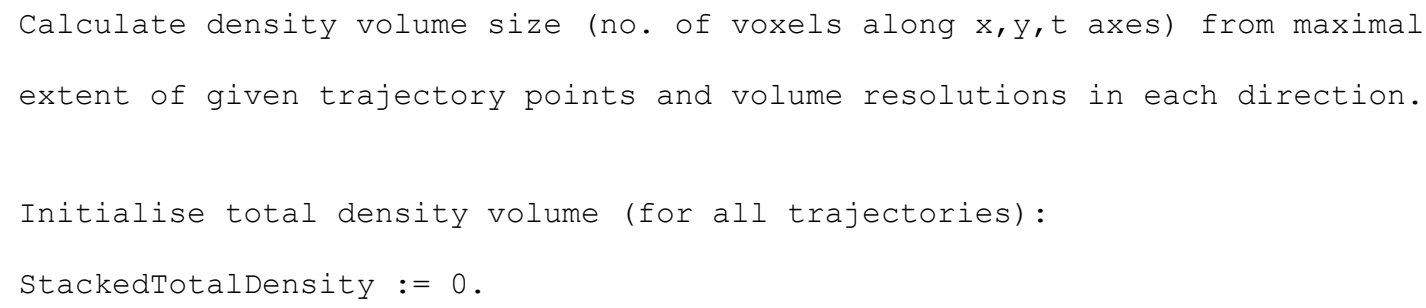

3. Inner loop 1 - this loop consists of steps 3. - 6. and runs through all points on current trajectory:

Subsampling to temporal resolution $\Delta t$ :

- If the next point on trajectory is more than $\Delta t$ away in dimension $t$, then assign this point to $(x 2, y 2, t 2)$.

- Else find the first next point on the trajectory that is more than $\Delta t$ away in dimension $t$ and assign this point to $(\mathrm{x} 2, \mathrm{y} 2, \mathrm{t} 2)$

4. Inner loop 2 - this loop consists of step 4. and runs through all temporal voxel levels (i.e. all horizontal layers) between two selected trajectory points $(\mathrm{x} 1, \mathrm{y} 1, \mathrm{t} 1)$ and $(\mathrm{x} 2, \mathrm{y} 2, \mathrm{t} 2)$.

\section{Stacking the densities:}

For each temporal voxel layer $t$ between temporal levels of points $(\mathrm{x} 1, \mathrm{y} 1, \mathrm{t} 1)$ and $(\mathrm{x} 2, \mathrm{y} 2, \mathrm{t} 2)$ do the following:

- Find the point on the line segment (x1,y1,t1)-(x2,y2,t2) at the centre of this particular voxel level =: central point CP. This point is found using linear interpolation between $(\mathrm{x} 1, \mathrm{y} 1, \mathrm{t} 1)$ and $(\mathrm{x} 2, \mathrm{y} 2, \mathrm{t} 2)$ at temporal level $t$. 


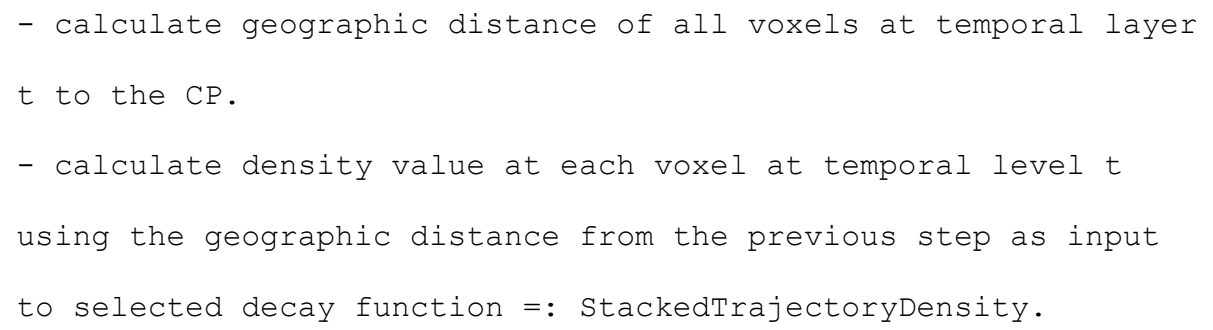

\section{References}

1. Andrienko G. and Andrienko N., 2010. A General Framework for Using Aggregation in Visual Exploration of Movement Data. The Cartographic Journal, 47(1), 22-40.

2. Benhamou S., 2011. Dynamic Approach to Space and Habitat Use Based on Biased Random Bridges. PLoS ONE, 6(1), e14592. 
3. Benhamou S. and Cornélis D., 2010. Incorporating Movement Behavior and Barriers to Improve Kernel Home Range Space Use Estimates. Journal of Wildlife Management, 74 (6), 1353-1360.

4. Benhamou S. and Riotte-Lambert L., 2012. Beyond the Utilization Distribution: Identifying home range areas that are intensively exploited or repeatedly visited. Ecological Modelling, 227(2012):112-116.

5. Börger L., Franconi N., De Michele G., Gantz A., Meschi F., Manica A., Lovari S., and Coulson T., 2006. Effects of sampling regime on the mean and variance of home range estimates. Journal of Animal Ecology, 75, 1393-1405.

6. Bouten W., Baaij E., Shamoun-Baranes J. and Camphuysen K.J., 2013. A flexible GPS tracking system for studying bird behaviour at multiple scales. Journal of Ornithology, 154:571-580.

7. Bridge E. S., Thorup K., Bowlin M. S., Chilson P. B., Diehl R. H., Flacron R. W., Hartl P., Roland K., Kelly J. F., Robinson W. D. and Wikelski M. 2011. Technology on the Move: Recent and Forthcoming Innovations for Tracking Migratory Birds. BioScience, 61:689-698.

8. Brillinger D. R., Preisler H. K, Ager A. A. and Kie J. G., 2004. An exploratory data analysis (EDA) of the paths of moving animals. Journal of Statistical Planning and Inference, 122, 43-63.

9. Brito, J. C., 2003. Seasonal Variation in Movements, Home Range, and Habitat Use by Male Vipera latastei in Northern Portugal. Journal of Herpetology, 37(1), 155-160.

10. Bullard F., 1999. Estimating the Home Range of an Animal: a Brownian Bridge Approach. MSc thesis. University of North Carolina at Chapel Hill. 
11. Callahan S. P., Callahan J. H., Scheidegger C. E. and Silva T. C., 2008. Direct Volume Rendering. Computing in Science \& Engineering, 2008(1), 88-92.

12. Camphuysen K, 2013, A historical ecology of two closely related gull species (Laridae): multiple adaptations to a man-made environment. PhD thesis, University of Groningen, The Netherlands.

13. Demšar U. and Virrantaus K., 2010. Space-time density of trajectories: exploring spatiotemporal patterns in movement data. International Journal of Geographical Information Science, 24(10), 1527-1542.

14. Downs J. A. and Horner M. W., 2009. A characteristic-hull based method for home range estimation. Transactions in GIS, 13(5-6):527-537.

15. Downs J. A., 2010, Time-Geographic Density Estimation for Moving Point Objects. In: Fabrikant S. I. et al. (eds): Proceedings of GIScience 2010. Lecture Notes in Computer Science, 6292:16-26.

16. Downs J. A., Horner M. W. and Tucker A. D., 2011. Time-geographic density estimation for home range analysis. Annals of GIS, 17(3):163-171.

17. Downs J. A. and Horner M. W., 2012. Analysing infrequently sampled animal tracking data by incorporating generalized movement trajectories with kernel density estimation. Computers, Environment and Urban Systems, 36:302-310.

18. Fieberg J., 2007. Kernel density estimators of home range: smoothing and the autocorrelation red herring. Ecology, 88(4), 1059-1066.

19. Getz W. M. and Wilmers C. C., 2004. A local nearest-neighbour convex-hull construction of home ranges and utilization distributions. Ecography, 27:489505. 
20. Getz W.M., Fortmann-Roe S., Cross P. C., Lyons A. J., Ryan S. J. and Wilmers C. C., 2007. LoCoH: Nonparametric Kernel Methods for Constructing Home Ranges and Utilization Distributions. PLoS ONE, 2, e207.

21. Ghisla A., 2009. Limitation and applicability of methods for home range estimation in respect to auto-ecological factors and data quality. MSc thesis (in Italian). Insubria University, Varese, Italy.

22. Hadwiger M., Sigg C., Scharsach H., Bühler K. and Gross M., 2005, Real-Time Ray-Casting and Advanced Shading of Discrete Isosurfaces. Eurographics 2005, 24 (3).

23. Hägerstrand T., 1970. What about people in regional science? Papers of the Regional Science Association, 24:7-21.

24. Hengl T., van Loon E. E., Shamoun-Baranes J. and Bouten W., 2008, Geostatistical Analysis of GPS Trajectory Data: Space-Time Densities. Proceedings of the $8^{\text {th }}$ International Symposium on Spatial Accuracy Assessment in natural Resources and Environmental Sciences, 17-24. Shanghai, June 2008.

25. Holden C., 2006. Inching Toward Movement Ecology. Science, 313, 779-782.

26. Horne J. S., Garton E. O, Krone S. M. and Lewis J. S., 2007a. Analyzing Animal Movements Using Brownian Bridges. Ecology, 88(9), 2354-2363.

27. Horne J. S., Garton E. O, Krone S. M. and Lewis J. S., 2007b. Analyzing Animal Movements Using Brownian Bridges - Appendix A: Derivation of Brownian bridge probability distribution. Ecological Archives, E088-142-A1.

28. Horne J. S., Garton E. O. and Sager-Fradkin K. A., 2007c. Correcting HomeRange Models for Observation Bias. Journal of Wildlife Management, 71(3), 996-1001. 
29. Isenberg T., Isenberg P., Chen J., Sedlmair M. and Möller T., 2013. A Systematic Review on the Practice of Evaluating Visualization. IEEE Transactions on Visualization and Computer Graphics, 19(12):2818-2827.

30. Kie J. G., Matthiopoulos J., Fieberg J., Powell R. A., Cagnacci F., Mitchell M. S., Gaillard J.-M. and Moorcroft P. R., 2010. The home-range concept: are traditional estimators still relevant with modern telemetry technology? Philosophical Transactions of Royal Society of London, Series B, Biological Sciences, 365, 2221-2231.

31. Kraak M-J., 2008. Geovisualization and Time - New Opportunities for the SpaceTime Cube. In: Dodge M., McDerby M. and Turner M. (eds), Geographic Visualization: Concepts, Tools and Applications. John Wiley \& Sons, Chichester, UK. 293-306, 2008.

32. Kranstauber B., Kays R., LaPoint S. D., Wikelski M. and Safi K., 2012. A dynamic Brownian bridge movement model to estimate utilization distributions for heterogeneous animal movement. Journal of Animal Ecology, 81:738-746.

33. Krisp J. M., Peters S and Burkert F., 2013. Visualizing Crowd Movement Patterns Using a Directed Kernel Density Estimation. Earth Observation of Global Changes (EOGC), Lecture Notes in Geoinformation and Cartography, 2013, 255-268.

34. Laver P. N. and Kelly M. J., 2008. A Critical Review of Home Range Studies. The Journal of Wildlife Management, 72(1), 290-298.

35. Long J. A. and Nelson T. A., 2011. Time Geography and Wildlife Home Range Delineation. The Journal of Wildlife Management, 76(2), 407-413. 
36. Long J. A. and Nelson T. A., 2012. A review of quantitative methods for movement data. International Journal of Geographical Information Science. Advanced online publication, doi:10.1080/13658816.2012.682578.

37. Manly B. F., McDonald L., Thomas D. L. McDonald T. L. and Erickson W. P., 2002. Resource Selection by Animals: Statistical Design and Analysis for Field Studies. $2^{\text {nd }}$ edition, Kluwer Academic Publishers.

38. Minnotte M. C., Sain S. R. and Scott D. W., 2008. Multivariate visualization by density estimation. In: Chen C., Härdle W and Unwin A. (eds.), Handbook of Data Visualization. Springer Handbooks of Computational Statistics, Springer Verlag, Berlin-Heidelberg, 390-413.

39. Nakaya T. and Yano K., 2010. Visualising crime clusters in a space-time cube: and exploratory data-analysis approach using space-time kernel density estimation and scan statistics. Transactions in GIS, 14(3):223-239.

40. Orians G. H. and Pearson N. E., 1979. On the theory of central place foraging. In: Horn D. J., Mitchell R. D. and Stairs G. R. (eds): Analysis of Ecological Systems. Ohio State University Press, Columbus, pp. 154-177.

41. Otis D. L. and White G. C., 1999. Autocorrelation of location estimates and the analysis of radiotracking data. Journal of Wildlife Management, 63(3), 10391044.

42. Riotte-Lambert L., Benhamou S. and Chamaillé-Jammes S., 2013. Periodicity analysis of movement recursions. Journal of Theoretical Biology, 317(2013):238-243.

43. Ropert-Coudert Y., Beaulieu M., Hanuise N. and Kato A., 2009. Diving into the world of biologging. Endangered Species Research, 10: 21-27. 
44. Rosenberg D. K. and McKelvey K.S., 1999. Estimation of habitat selection for central-place foraging animals. The Journal of Wildlife Management, 63(3), 1028-1038.

45. Seaman D. E. and Powell R. A., 1996. An evaluation of the accuracy of kernel density estimators for home range analysis. Ecology, 77(7), 2075-2085.

46. Shamoun-Baranes J., van Loon E., van Gasteren H., van Belle J., Bouten W. and Buurma L., 2006. A comparative analysis of the influence of weather on the flight altitudes of birds. Bulletin of the American Meteorology Society, 87: 4761.

47. Shamoun-Baranes J., van Loon E. E., Purves R. S., Speckmann B., Weiskopf D. and Camphuysen C. J., 2012. Analysis and visualization of animal movement. Biology Letters, 8(1): 6-9.

48. Shamoun-Baranes J., Bom R., van Loon E.E., Ens B.J., Oosterbeek K. and Bouten W., 2012. From Sensor Data to Animal Behaviour: An Oystercatcher Example. PLoS ONE, 7:e37997.

49. Scheepens R., Willems N., van de Wetering H. and van Wijk, J.J., 2011. Interactive Visualization of Multivariate Trajectory Data with Density Maps. IEEE Pacific Visualisation Symposium 2001, 147-154.

50. Silverman B.W., 1986. Density estimation for statistics and data analysis. Chapman and Hall, New York.

51. Stephens D. W., Brown J. S. and Ydenberg R. C., 2007. Foraging: Behaviour and Ecology. University of Chicago Press, Chicago.

52. Van Deelen T. R., Campa H. III, Hamady M. and Haufler, J. B., 1998. Migration and seasonal range dynamics of deer using adjacent deeryards in northern Michigan. Journal of Wildlife Management, 62(1), 205-213. 
53. Walter D. W., Beringer J., Hansen L. P, Fischer J. W., Millspaugh J. J. and Vercauteren K. C., 2011. Factors affecting space use overlap by white-tailed deer in an urban landscape. International Journal of Geographical Information Science, 25(3), 379-392.

54. Wand M. P. and Jones M. C., 1993. Comparison of Smoothing Parametrizations in Bivariate Kernel Density Estimation. Journal of American Statistical Association, 88(422): 520-528.

55. Worton B. J., 1987. A review of models of home range for animal movement. Ecological Modelling, 38, 277-298.

56. Worton B. J., 1989. Kernel Methods for Estimating the Utilization Distribution in Home-Range Studies. Ecology, 70(1), 164-168.

57. Wu Y., Qu H., Chung K.-K., Chan M.-Y. and Zhou H., 2010. Quantitative Effectiveness Measures for Direct Volume Rendered Images, IEEE Pacific Visualisation Symposium 2010. 1-8. 


\section{Figure captions}

Fig. 1. Two- (a, b, c, d) and three-dimensional kernels (e, f, g, h) for trajectories. Two-dimensional kernels produce density surfaces, three-dimensional kernels produce volumes in geo-time space. Panels a and e show point-based kernels that do not consider temporal dimension of trajectory points but instead use each point as an independent measurements in a point data set. Panels b-d produce line-segment kernels: one kernel for each line segment of the trajectory and these kernels are then summed up along the entire line: kernels from two consecutive line segments overlap each other. Panels f-h produce polyline kernels: one kernel for the entire trajectory (and not a separate kernel for each line segment). Distance from each voxel and trajectory in polyline kernel in panel $\mathrm{f}$ (shown in kernel with a dashed grey line) is measured perpendicularly to the trajectory and can thus be in any 3D direction. Distance from each voxel and trajectory in polyline kernels in panels g-h is always measured in horizontal direction (at a constant moment in time) and is calculated as 2D distance. Although stacked kernels in g-h produce a visually similar density volume to smooth kernel in panel $f$, they are not a special case of $f$, since they are calculated differently.

Fig. 2. Four different decays shown as density volumes on one artificial trajectory and corresponding decay functions: a) linear, b) bisquare, c) Gaussian and d) Brownian. Linear and bisquare decay functions reach value 0 at distance $h$ from trajectory, where $h$ denotes bandwidth. Height $a$ is determined so that the functions satisfy the requirement of their respective rotated surfaces being 2D probability distributions (i.e. volume under rotated surface has to be equal to 1). Gaussian decay function is the 
normal distribution with the mean centred on trajectory (i.e. $\mu=0$ ) and variance $\sigma^{2}$ dependent on bandwidth $h$, but constant the for entire trajectory. Brownian decay is based on the Brownian bridges approach, where at each level in the space-time cube we use a Gaussian decay function with constant mean as above $(\mu=0)$, but where variance depends on the length of the current line segment $(T)$ and the position of the current voxel layer on that particular line segment $(t)$. The closer the voxel position is to the ends of the line segment, the steeper and narrower is the shape of the distribution.

Fig. 3. Comparison of $2 \mathrm{D}$ and $3 \mathrm{D}$ densities on two simulated sets of trajectories. Each set of trajectories includes four trajectories. Panel (a) shows the space-time cube of trajectory set A, representing a spatial-only hotspot and panel (b) the space time cube of trajectory set B, representing a spatio-temporal hotspot. Panel (c) shows the spatial distributions of points in each set: these distributions are identical for both sets of trajectories. (d) is a map of the $2 \mathrm{D}$ density of trajectories in each set: these two densities are also identical for both sets of trajectories. However, (e) the stacked space-time density volume of trajectory set A and (f) the stacked space-time density volume of trajectory set B present a different pattern and enable visual distinction between two different types of movement.

Fig. 4. Comparing space-time (ST) density [13] vs. stacked densities. We use the same artificial trajectory data sets to calculate ST and stacked density fields. Each of these fields is then displayed as density volume using the same volume rendering method. In statistics, density fields are often compared directly [20] using various error and other measures (first purple arrow). We cannot do this, since our two density 
fields treat volume dimensions differently [54]. In volumetric visualisation, different volumes are compared through quantification of efficiency [57] of their respective volume rendering methods (second purple arrow). This is not applicable in our case, since we use the same rendering for both volumes. Instead, we adopt two frequently applied evaluation methods from information visualisation [29], algorithm performance (left green arrow) and qualitative inspection (right green arrow).

Fig. 5. Space-time density [13] for a) a spatial-only hotspot (case A) and b) spatiotemporal hotspot (case B). Densities are shown with identical volume rendering parameters as stacked densities in panels 3e) and 3f) respectively.

Fig. 6. Geographic positions of a) bird 298 and b) bird 311, covering a time period of 30 days (June 2010). c) Location of nests on Texel island and 150m buffers around each individual nest that were used to compensate for central-place foraging behaviour of lesser black backed gulls by d) removing all trajectory points within the $150 \mathrm{~m}$ of the nest (bird 311 in this example). In the background is the land use map of this area of the Netherlands.

Fig. 7. Space-time density for bird 298 calculated using four different decay types: a) linear decay, b) bisquare decay, c) Gaussian decay and d) decay using the Brownian bridges approach. The $x$ and $y$ axis show easting and northing in metres and the $z$ axis time in seconds $(24 \mathrm{~h}=86400 \mathrm{~s})$. In this example, the nest is already removed from the analysis. 
Fig. 8. Correction for central-place foraging behaviour of lesser black backed gulls. Figure shows two space-time densities for bird 311, where in a) all points were taken into account and in b) points within the $150 \mathrm{~m}$ buffer around the nest were removed prior to density calculation.

Fig. 9. Space-time density for bird 298 using Gaussian decay, shown from different angles with volume rendering in a) and b), c) from the south, d) from the top and e) with an isosurface. The area around the nest is identifiable as a temporal column that spans the entire height of the volume (24h). There is an additional smaller temporal column, south-east from the nest, which represents a spatio-temporal hot spot. This is a place that the bird visited frequently, mostly in the mornings and stayed there for a while. This is best seen in the view from the front, c) and in the isosurface displays e), where this hot spot is one uninterrupted dense area. Geographical location of this hot spot can be identified in the top view (d), where it is shown with an arrow for clarity.

Fig. 10. Space-time density for bird 311 using Gaussian decay, shown with volume rendering in a) and b), c) from the east, d) from the top and e) with an isosurface. The temporal column that identifies the area around the nest is clearly identifiable. There is another area that is visited often, indicated with an arrow in the top view d), which represents a spatial only hot spot, i.e. an area that the bird visits often, but where it does not stay for long. This is most clearly identifiable in the isosurface, where this area consists of several floating high density areas, located at different heights and therefore at different moments in time. 


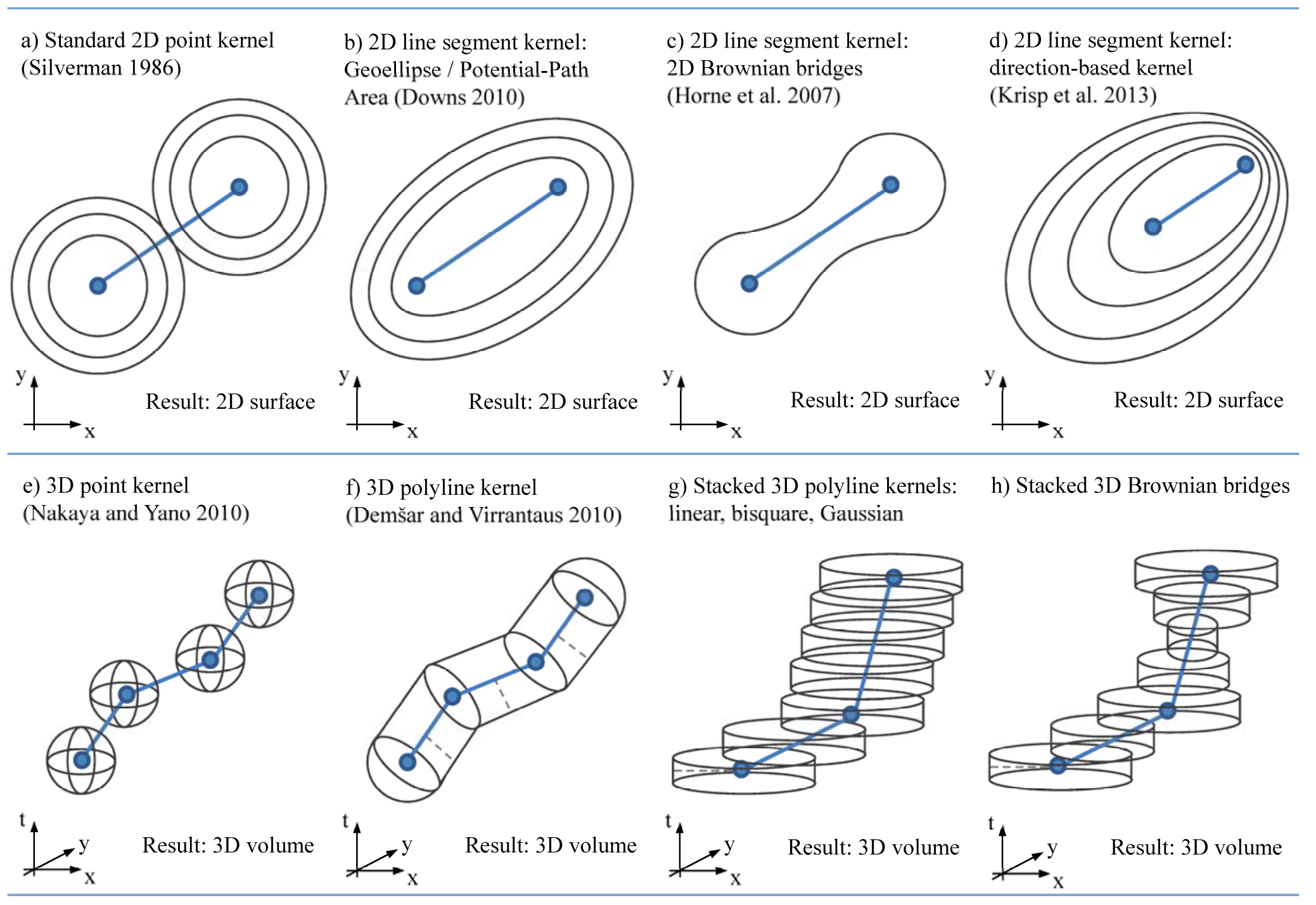

Fig. 1. Two- (a, b, c, d) and three-dimensional kernels (e, f, g, h) for trajectories. Two-dimensional kernels produce density surfaces, threedimensional kernels produce volumes in geo-time space. Panels a and e show point-based kernels that do not consider temporal dimension of trajectory points but instead use each point as an independent measurements in a point data set. Panels b-d produce line-segment kernels: one kernel for each line segment of the trajectory and these kernels are then summed up along the entire line: kernels from two consecutive line segments overlap each other. Panels f-h produce polyline kernels: one kernel for the entire trajectory (and not a separate kernel for each line $50 / 63$ 
Manuscript accepted to Geoinformatica, 2014

segment). Distance from each voxel and trajectory in polyline kernel in panel $\mathrm{f}$ (shown in kernel with a dashed grey line) is measured perpendicularly to the trajectory and can thus be in any 3D direction. Distance from each voxel and trajectory in polyline kernels in panels g-h is always measured in horizontal direction (at a constant moment in time) and is calculated as 2D distance. Although stacked kernels in g-h produce a visually similar density volume to smooth kernel in panel f, they are not a special case of f, since they are calculated differently. 
a)

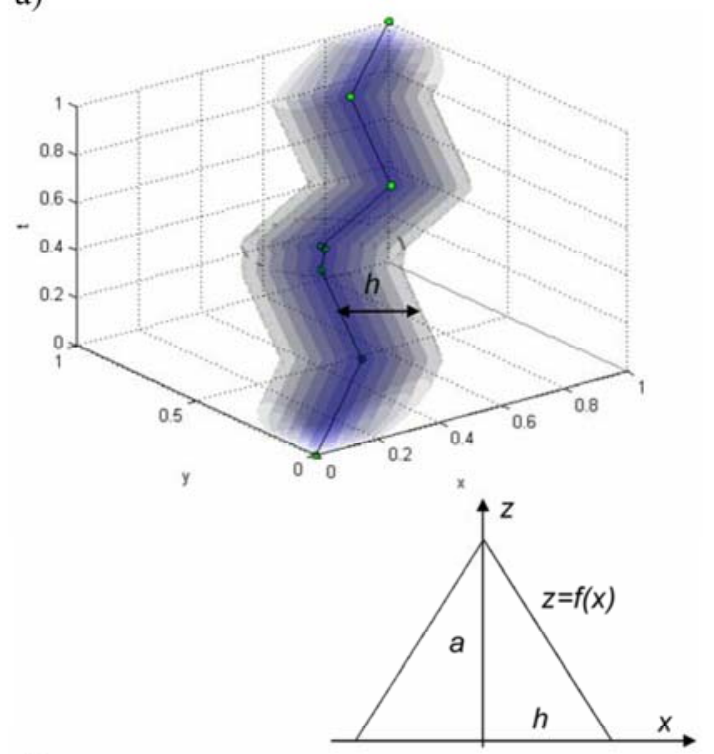

c)

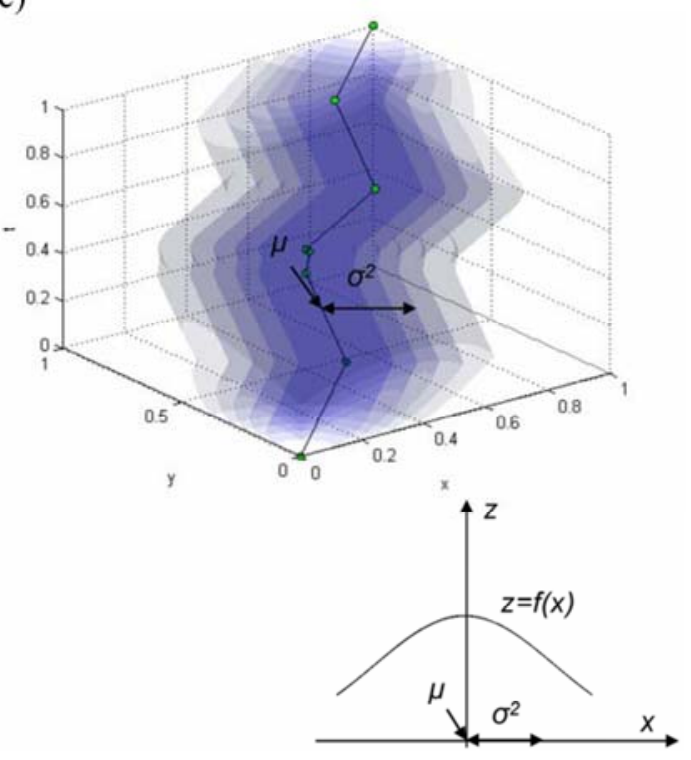

b)

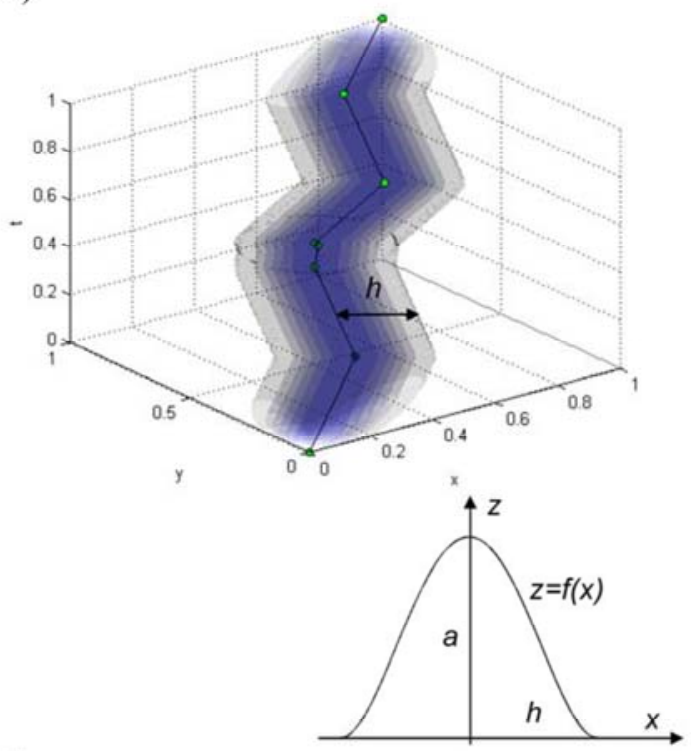

d)

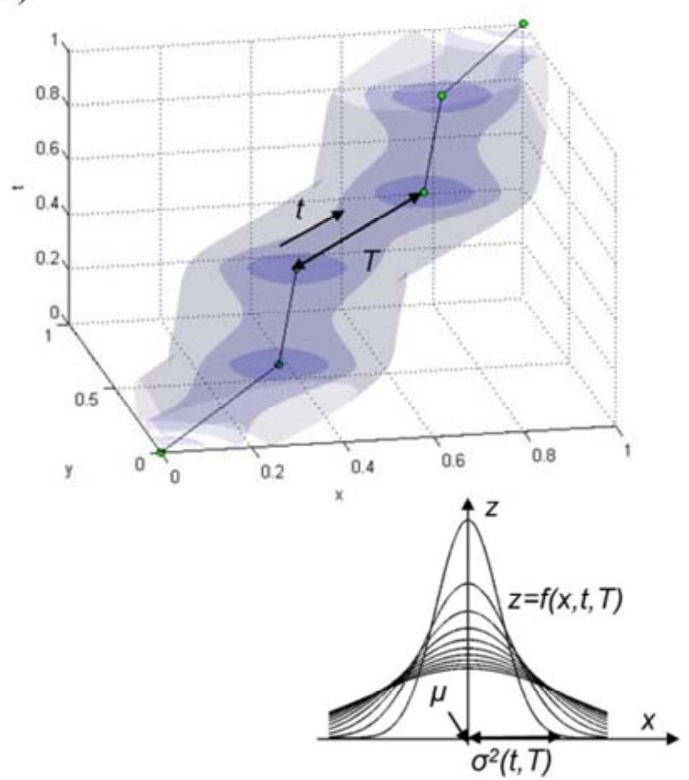

Fig. 2. Four different decays shown as density volumes on one artificial trajectory and corresponding decay functions: a) linear, b) bisquare, c) Gaussian and d) Brownian. Linear and bisquare decay functions reach value 0 at distance $h$ from trajectory, where $h$ denotes bandwidth. Height $a$ is determined so that the functions satisfy the requirement of their respective rotated surfaces being 2D probability distributions (i.e. volume under rotated surface has to be equal to 1). Gaussian decay function is the normal distribution with the mean centred on trajectory (i.e. $\mu=0$ ) and variance $\sigma^{2}$ dependent on bandwidth $h$, but constant the for entire trajectory. Brownian decay is based on the Brownian bridges approach, where at each level in the space-time cube we use a Gaussian decay function with constant mean as above $(\mu=0)$, but where variance depends on the length of the current line segment $(T)$ and the position of the current voxel layer on that particular line segment $(t)$. The closer the voxel position is to the ends of the line segment, the steeper and narrower is the shape of the distribution. 
a)

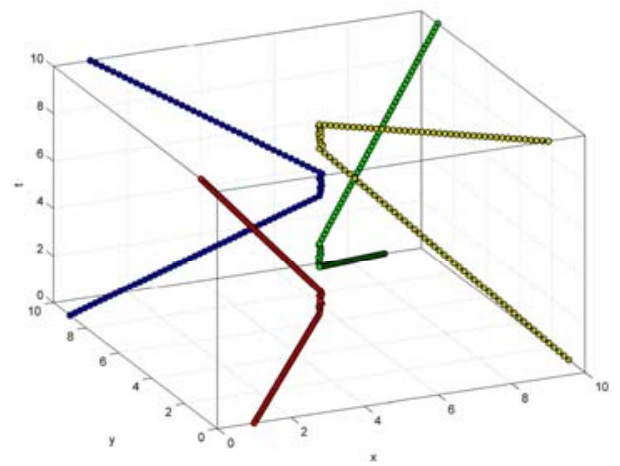

c)

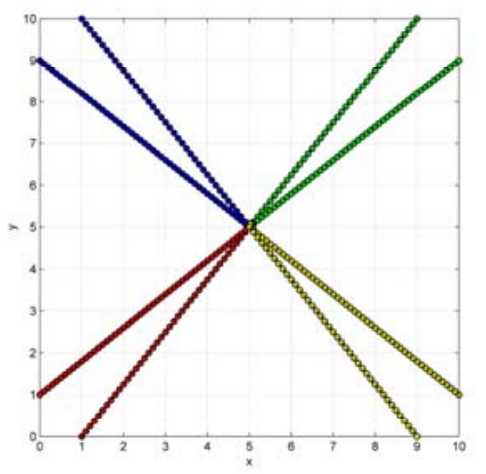

e)

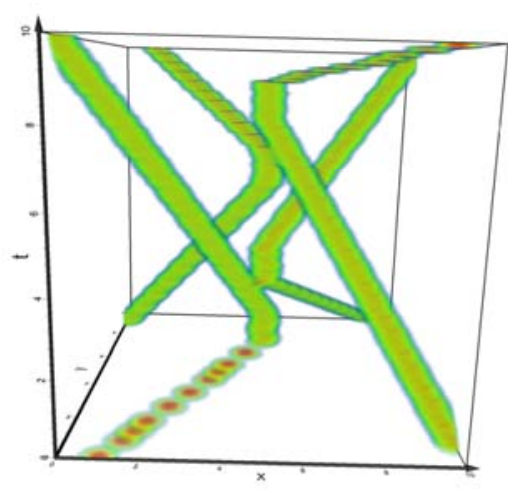

b)

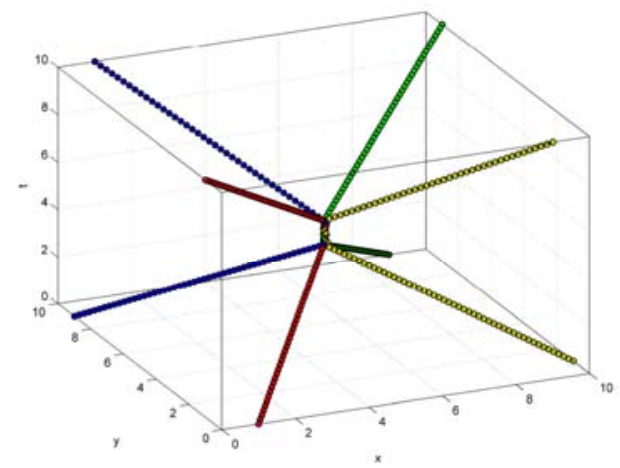

d)

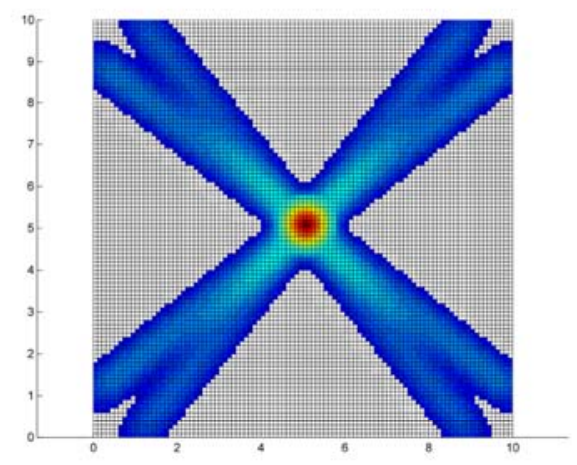

f)

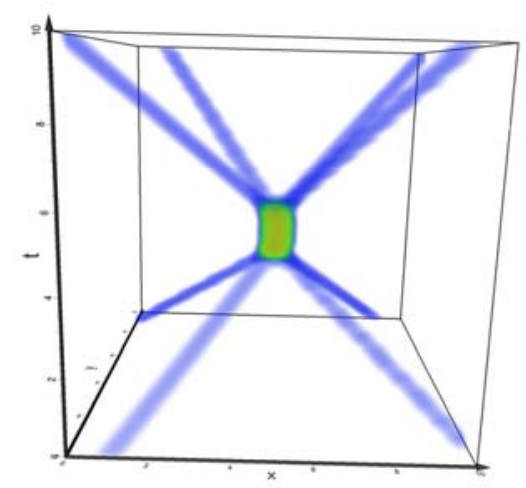

Fig. 3. Comparison of 2D and 3D densities on two simulated sets of trajectories. Each set of trajectories includes four trajectories. Panel (a) shows the space-time cube of trajectory set A, representing a spatial-only hotspot and panel (b) the space time cube of trajectory set B, representing a spatio-temporal hotspot. Panel (c) shows the spatial distributions of points in each set: these distributions are identical for both sets of trajectories. (d) is a map of the 2D density of trajectories in each set: these two densities are also identical for both sets of trajectories. However, (e) the stacked space-time density volume of trajectory set A and (f) the stacked space-time density volume of trajectory set B present a different pattern and enable visual distinction between two different types of movement. 


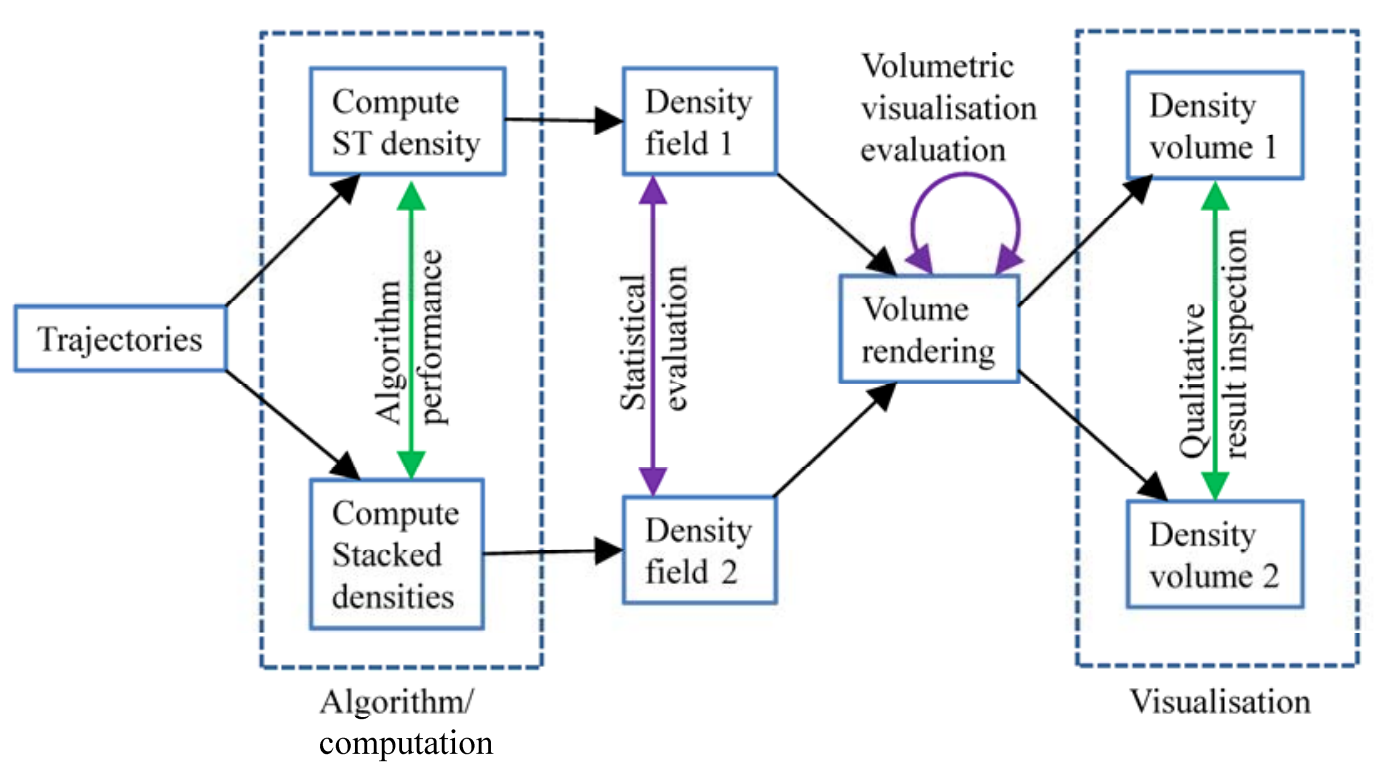

Fig. 4. Evaluation process of space-time (ST) density vs. stacked densities. We use the same artificial trajectory data sets to calculate ST and stacked density fields. Each of these fields is then displayed as density volume using the same volume rendering method. In statistics, density fields are often compared directly [20] using various error and other measures (first purple arrow). We cannot do this, since our two density fields treat volume dimensions differently [54]. In volumetric visualisation, different volumes are compared through quantification of efficiency [57] of their respective volume rendering methods (second purple arrow). This is not applicable in our case, since we use the same rendering for both volumes. Instead, we adopt two frequently applied evaluation methods from information visualisation [29], algorithm performance (left green arrow) and qualitative inspection (right green arrow). 
a)

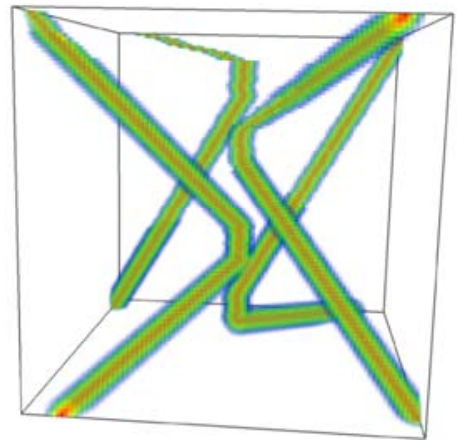

b)

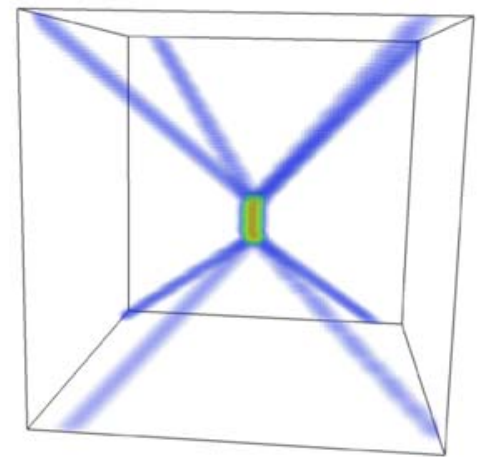

Fig. 5. Space-time density [13] for a) a spatial-only hotspot (case A) and b) spatiotemporal hotspot (case B). Densities are shown with identical volume rendering parameters as stacked densities in panels 3e) and 3f) respectively. 
a)

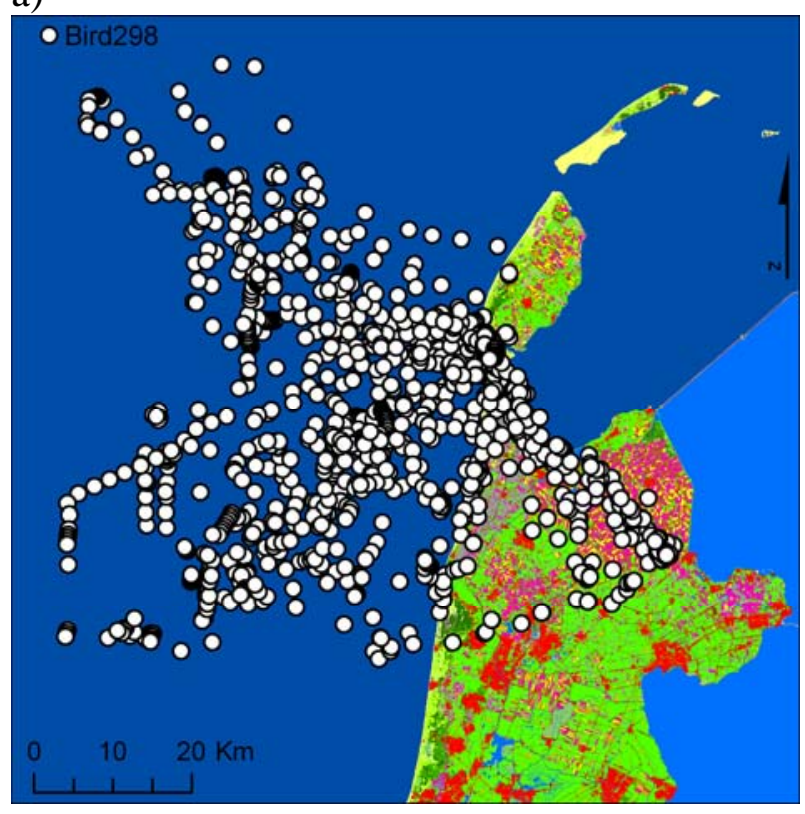

c)

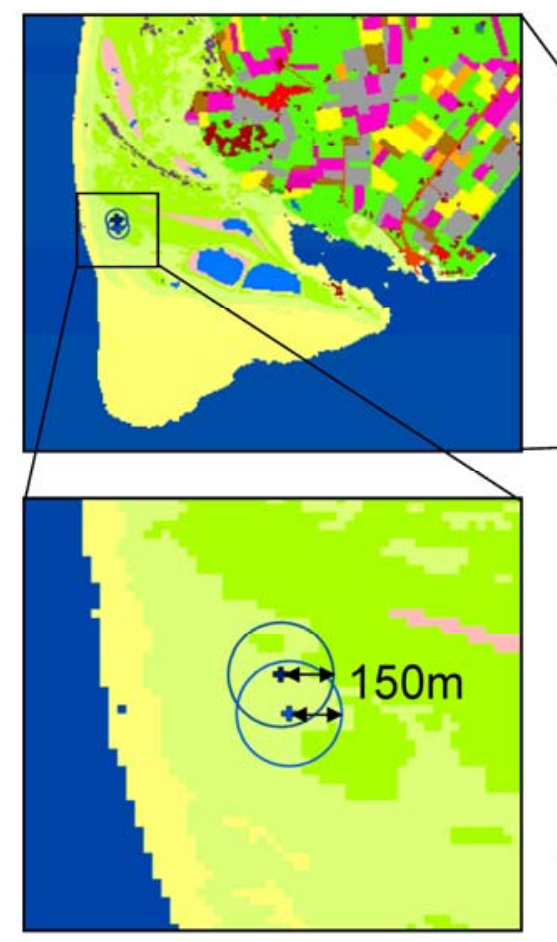

b)

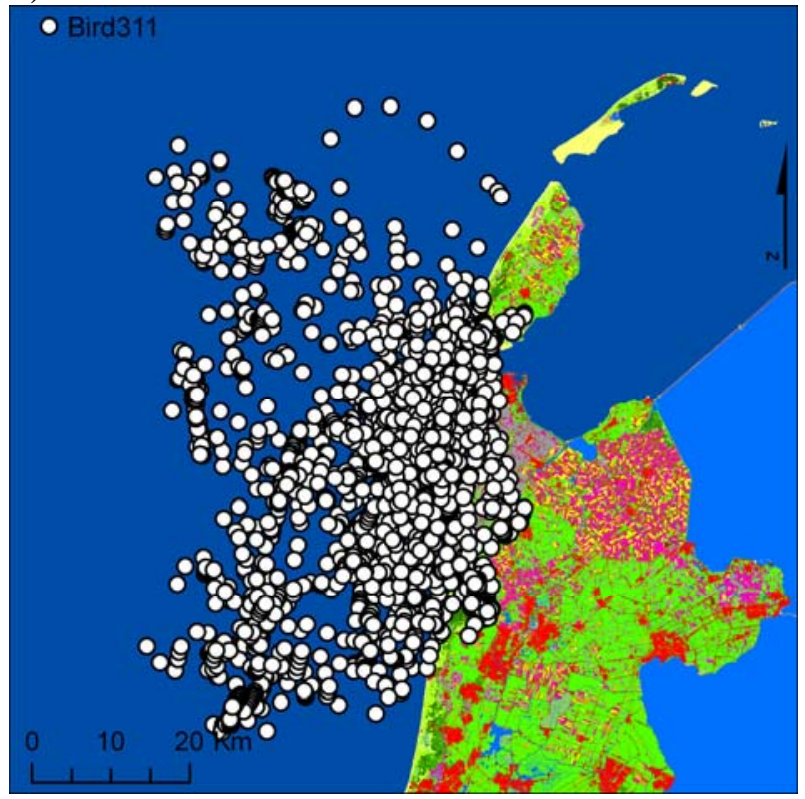

d)
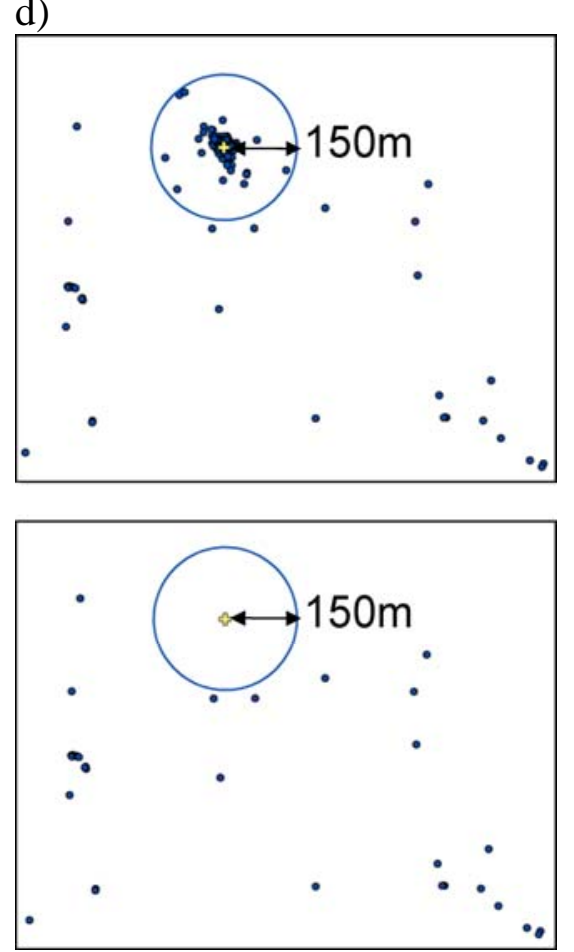

Fig. 6. Geographic positions of a) bird 298 and b) bird 311, covering a time period of 30 days (June 2010). c) Location of nests on Texel island and $150 \mathrm{~m}$ buffers around each individual nest that were used to compensate for central-place foraging behaviour of lesser black backed gulls by d) removing all trajectory points within the $150 \mathrm{~m}$ of the nest (bird 311 in this example). In the background is the land use map of this area of the Netherlands. 
a)

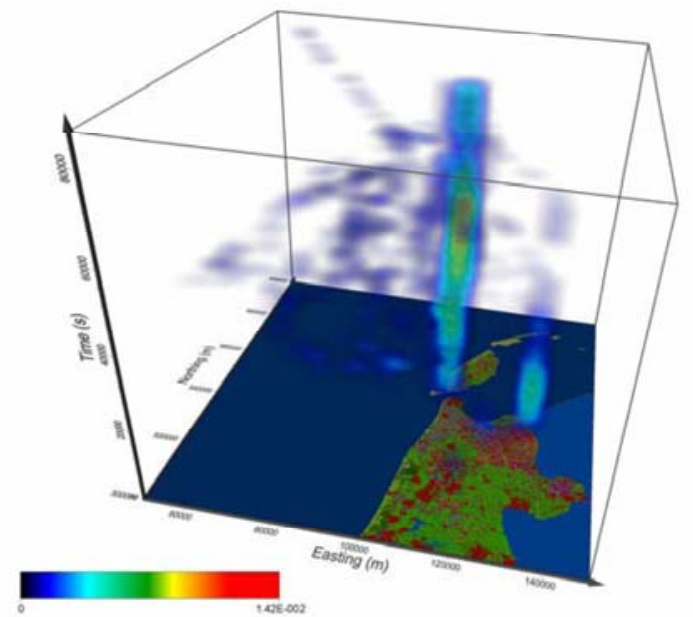

c)

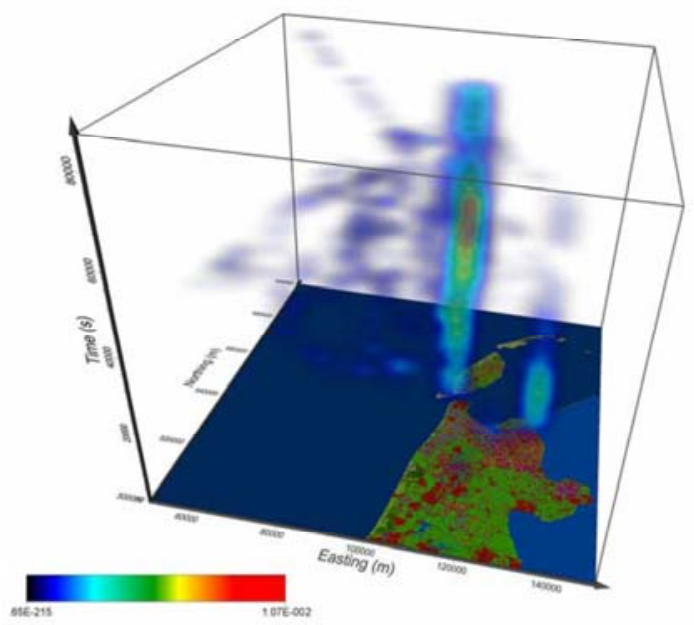

b)

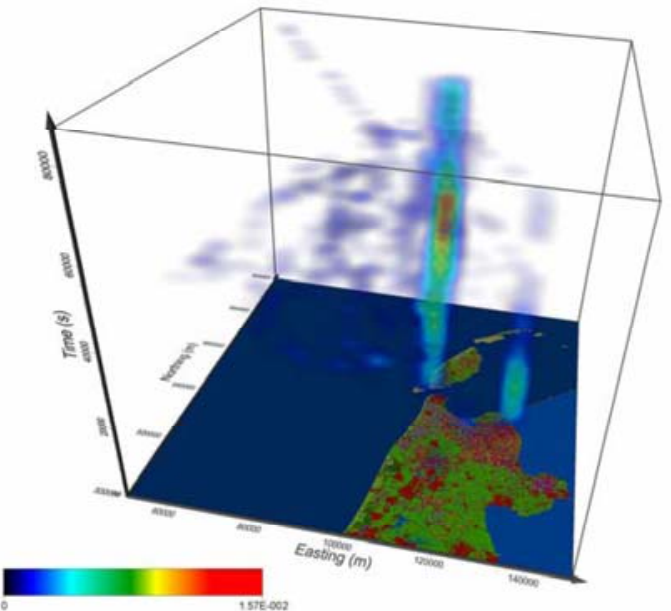

d)

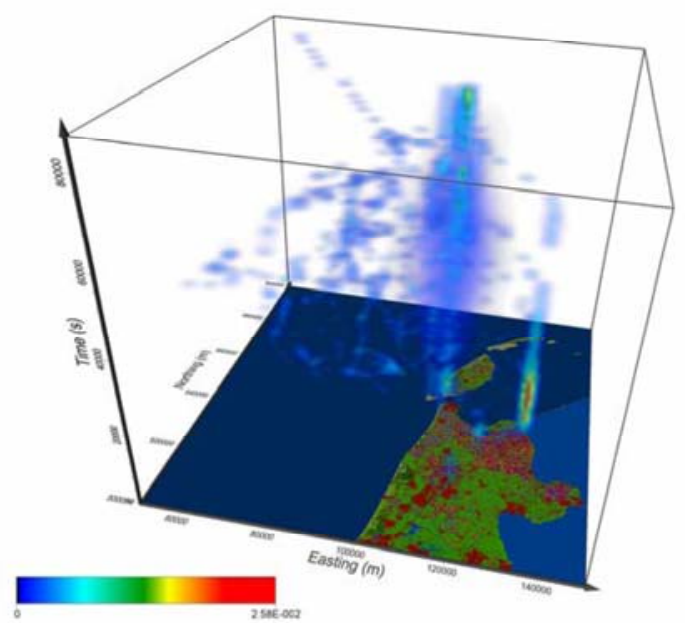

Fig. 7. Space-time density for bird 298 calculated using four different decay types: a) linear decay, b) bisquare decay, c) Gaussian decay and d) decay using the Brownian bridges approach. The $x$ and $y$ axis show easting and northing in metres and the $z$ axis time in seconds $(24 \mathrm{~h}=86400 \mathrm{~s})$. In this example, the nest is already removed from the analysis. 
a)

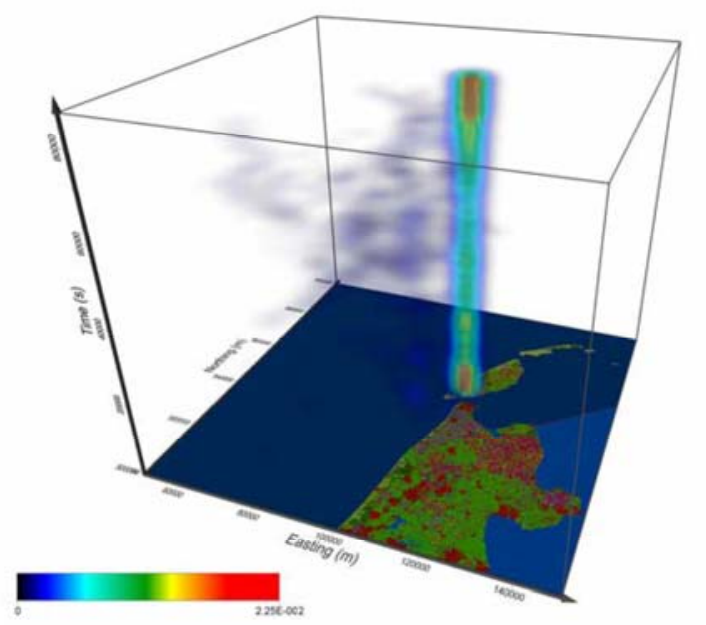

b)

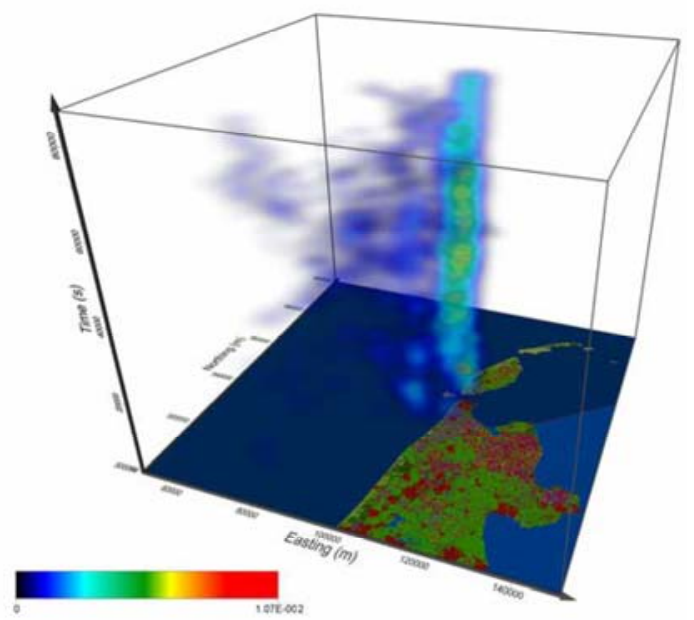

Fig. 8. Correction for central-place foraging behaviour of lesser black backed gulls. Figure shows two space-time densities for bird 311, where in a) all points were taken into account and in b) points within the $150 \mathrm{~m}$ buffer around the nest were removed prior to density calculation. 
a)

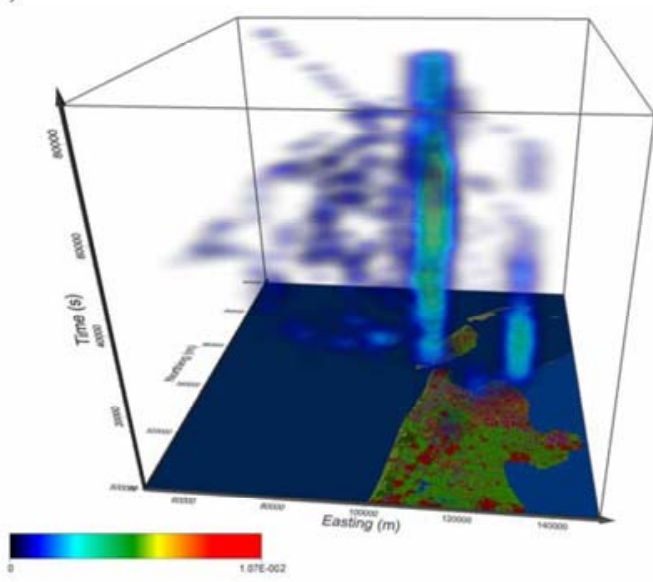

c)

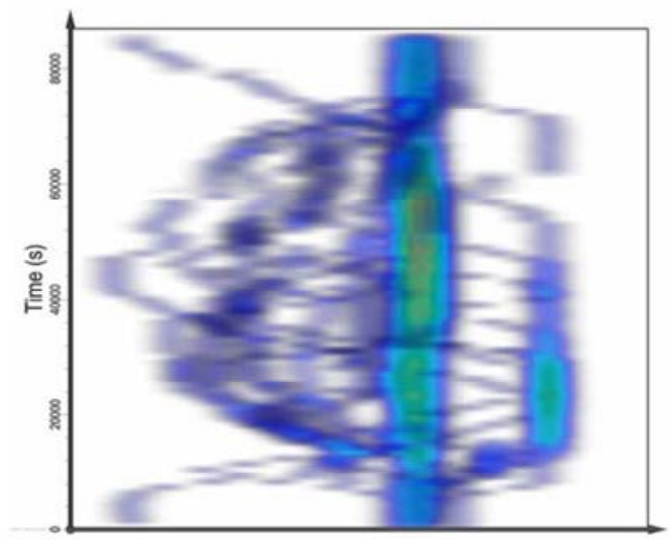

e)

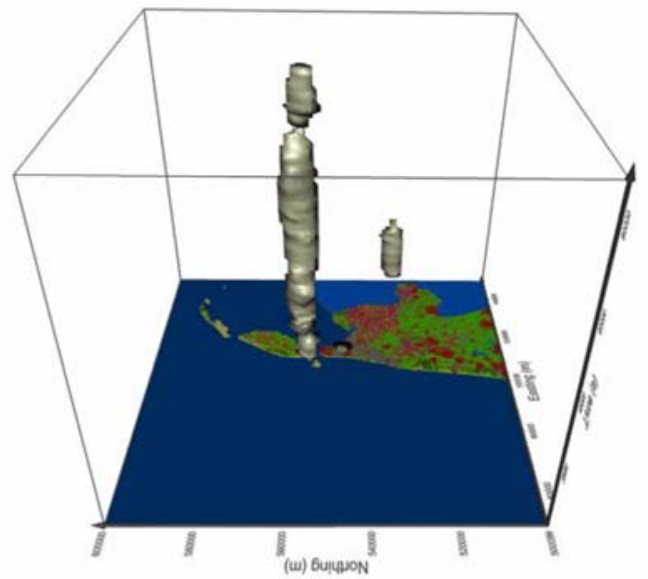

b)

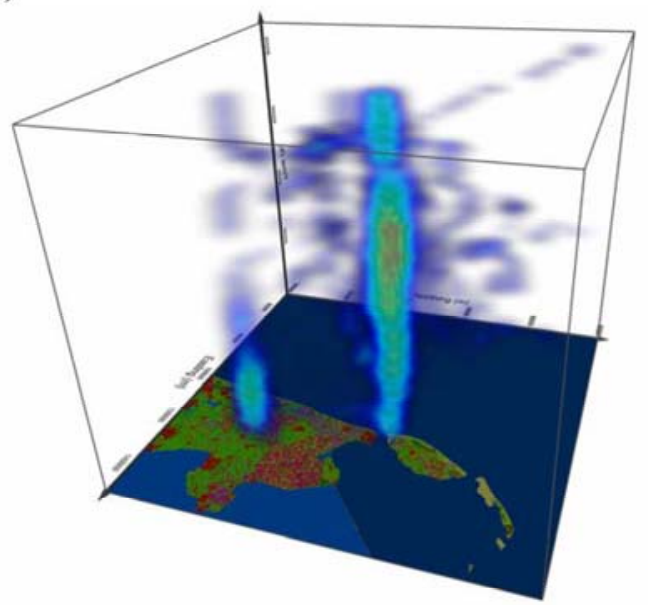

d)
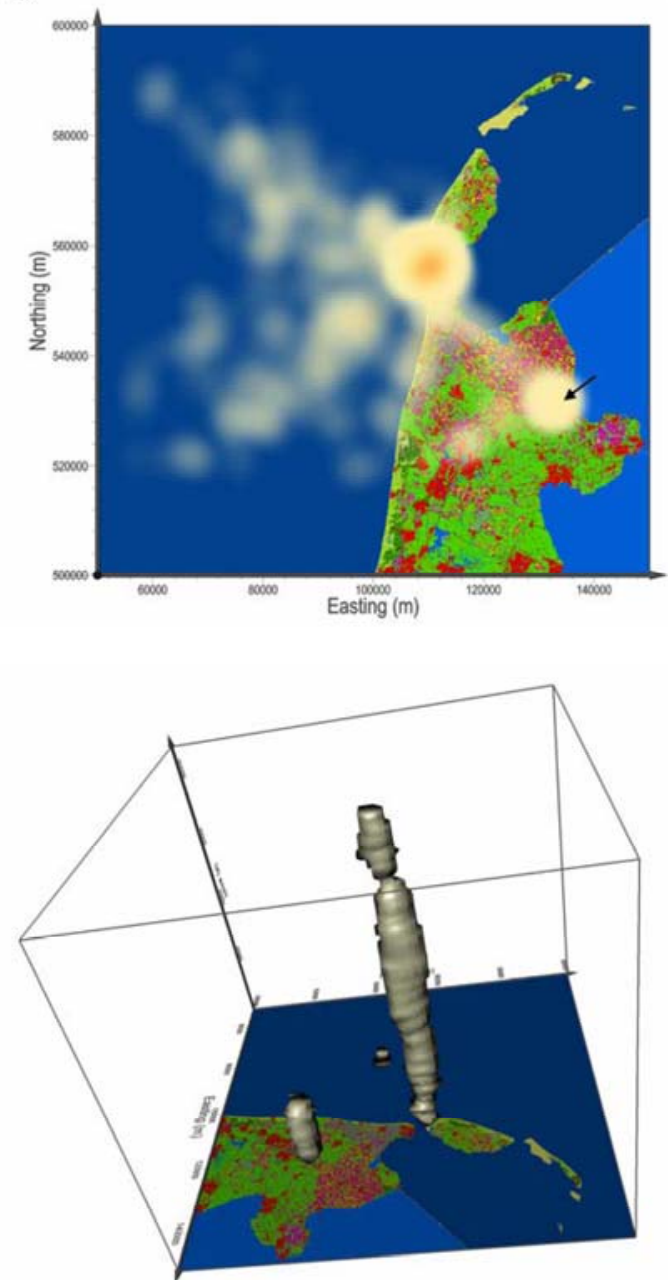

Fig. 9. Space-time density for bird 298 using Gaussian decay, shown from different angles with volume rendering in a) and b), c) from the south, d) from the top and e) with an isosurface. The area around the nest is identifiable as a temporal column that spans the entire height of the volume (24h). There is an additional smaller temporal column, south-east from the nest, which represents a spatio-temporal hot spot. This is a place that the bird visited frequently, mostly in the mornings and stayed there for a while. This is best seen in the view from the front, c) and in the isosurface displays e), where this hot spot is one uninterrupted high density area. Geographical location of 
Manuscript accepted to Geoinformatica, 2014

this hot spot can be identified in the top view (d), where it is shown with an arrow for clarity. 
a)

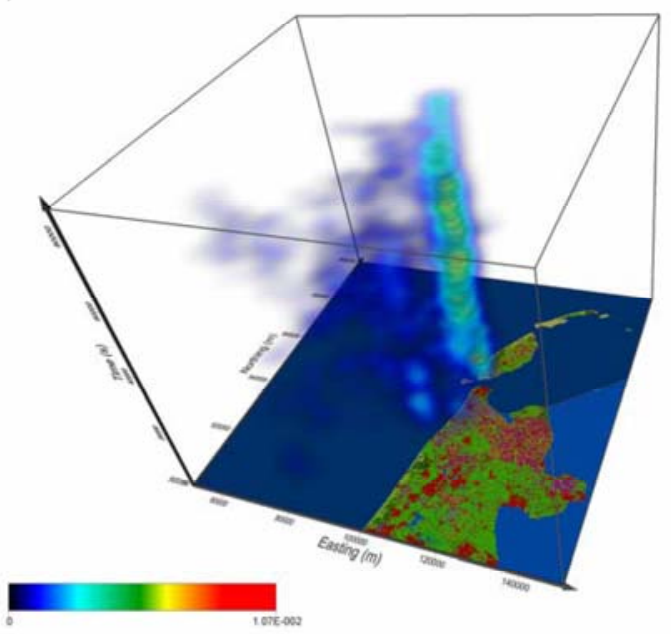

c)

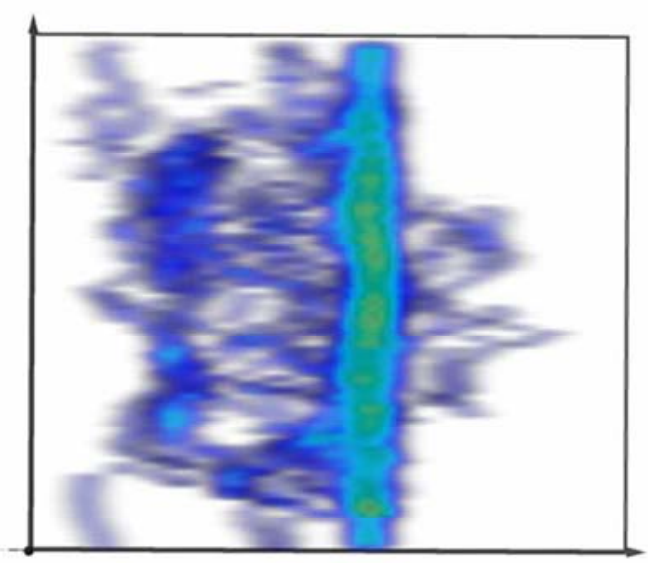

e)

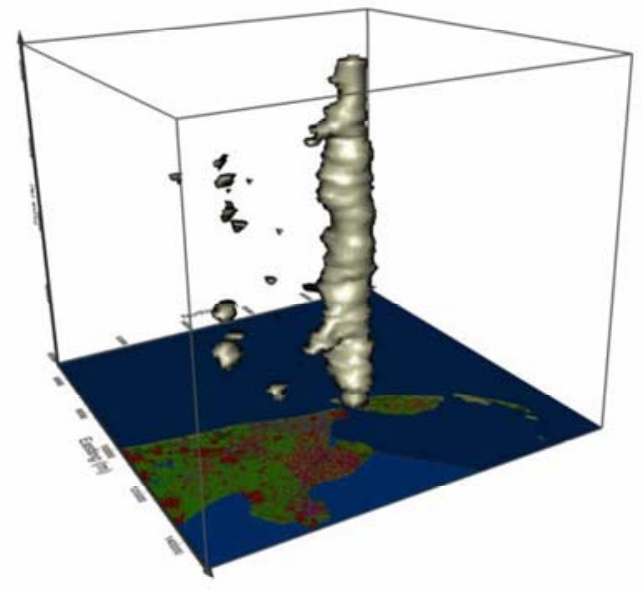

b)

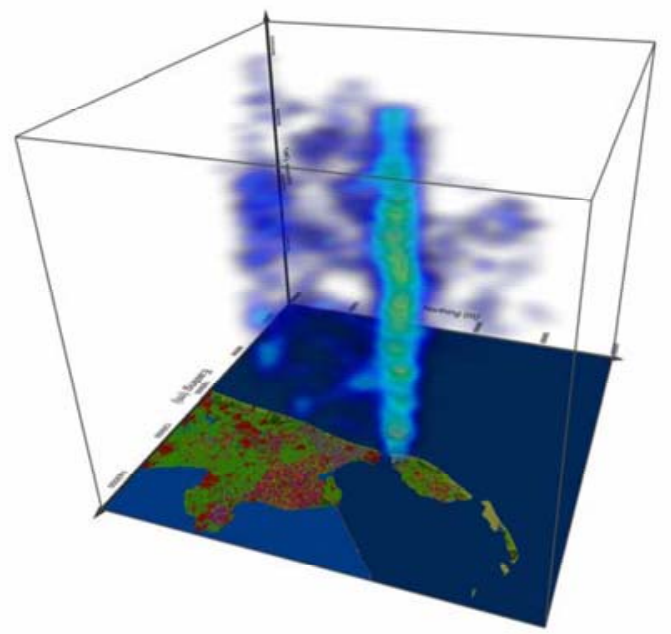

d)
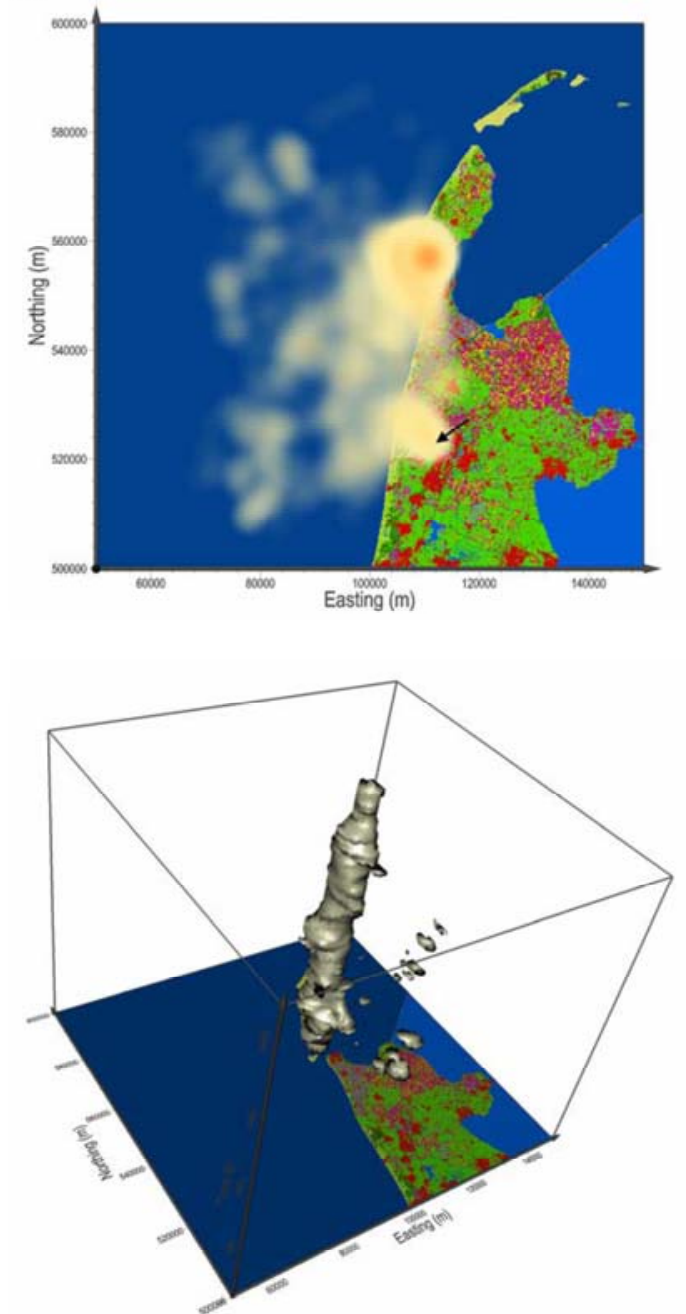

Fig. 10. Space-time density for bird 311 using Gaussian decay, shown with volume rendering in a) and b), c) from the east, d) from the top and e) with an isosurface. The temporal column that identifies the area around the nest is clearly identifiable. There is another area that is visited often, indicated with an arrow in the top view d), which represents a spatial only hot spot, i.e. an area that the bird visits often, but where it does not stay for long. This is most clearly identifiable in the isosurface, where this 
area consists of several floating high density areas, located at different heights and therefore at different moments in time. 
Table 1. Algorithm performance of the two density algorithms: space-time density [13] and stacked densities.

\begin{tabular}{lllllrrrr}
\hline $\begin{array}{l}\text { Max traj. } \\
\text { length }\end{array}$ & $\begin{array}{l}\text { Data set } \\
\text { range }\end{array}$ & Volume size & $\begin{array}{l}\text { Voxel } \\
\text { size }\end{array}$ & \multicolumn{2}{l}{$\begin{array}{l}\text { Ker } \\
\text { nel }\end{array}$} & \multicolumn{2}{c}{$\begin{array}{c}\text { Processing times - } \\
\text { space-time density [13] }\end{array}$} & \multicolumn{2}{c}{$\begin{array}{c}\text { Processing times - } \\
\text { stacked densities }\end{array}$} \\
\hline 50 & {$[0,10]^{3}$} & $10 \times 10 \times 10$ & 1 & 3 & $3.90 \mathrm{~s}$ & $4.05 \mathrm{~s}$ & $0.04 \mathrm{~s}$ & $0.02 \mathrm{~s}$ \\
50 & {$[0,10]^{3}$} & $100 \times 100 \times 100$ & 0.1 & 3 & $2816.27 \mathrm{~s}$ & $2966.29 \mathrm{~s}$ & $12.89 \mathrm{~s}$ & $13.28 \mathrm{~s}$ \\
100 & {$[0,10]^{3}$} & $10 \times 10 \times 10$ & 1 & 3 & $7.68 \mathrm{~s}$ & $8.45 \mathrm{~s}$ & $0.06 \mathrm{~s}$ & $0.03 \mathrm{~s}$ \\
100 & {$[0,10]^{3}$} & $100 \times 100 \times 100$ & 0.1 & 3 & $5168.51 \mathrm{~s}$ & $5478.07 \mathrm{~s}$ & $15.36 \mathrm{~s}$ & $14.84 \mathrm{~s}$ \\
\hline
\end{tabular}

